\title{
Effects of exercise therapy in patients with acute low back pain: a systematic review of systematic reviews
}

\author{
Marc Karlsson 1,2, Anna Bergenheim²,3,4, Maria E. H. Larsson 2,4, Lena Nordemann ${ }^{2,4}$, Maurits van Tulder ${ }^{5,6}$ and \\ Susanne Bernhardsson ${ }^{2,4^{*}}$ (D)
}

\begin{abstract}
Background: Acute low back pain is associated with pain and disability, but symptoms are often self-healing. The effectiveness of exercise therapy for acute low back pain remains uncertain with conflicting evidence from systematic reviews. The aim of this systematic review of systematic reviews was to assess the overall certainty of evidence for the effects of exercise therapy, compared with other interventions, on pain, disability, recurrence, and adverse effects in adult patients with acute low back pain.

Methods: PubMed, the Cochrane library, CINAHL, PEDro, Open Grey, Web of Science, and PROSPERO were searched for systematic reviews of randomized controlled trials. Methodological quality was assessed independently by two authors using AMSTAR. Meta-analyses were performed if possible, using data from the original studies. Data for pain, disability, recurrence, and adverse effects were analyzed. Certainty of evidence was assessed using GRADE.

Results: The searches retrieved 2602 records, of which 134 publications were selected for full-text screening. Twenty-four reviews were included, in which 21 randomized controlled trials $(n=2685)$ presented data for an acute population, related to 69 comparisons. Overlap was high, 76\%, with a corrected covered area of 0.14 . Methodological quality varied from low to high. Exercise therapy was categorized into general exercise therapy, stabilization exercise, and McKenzie therapy. No important difference in pain or disability was evident when exercise therapy was compared with sham ultrasound, nor for the comparators usual care, spinal manipulative therapy, advice to stay active, and educational booklet. Neither McKenzie therapy nor stabilization exercise yielded any important difference in effects compared with other types of exercise therapy. Certainty of evidence varied from very low to moderate.
\end{abstract}

(Continued on next page)

\footnotetext{
* Correspondence: susanne.bernhardsson@vgregion.se

${ }^{2}$ Department of Health and Rehabilitation, Unit of Physiotherapy, The

Sahlgrenska Academy, Institute of Neuroscience and Physiology, University of Gothenburg, Gothenburg, Sweden

${ }^{4}$ Region Västra Götaland, Research and Development Primary Health Care, Kungsgatan 12, 6th floor, SE-412 19 Gothenburg, Sweden

Full list of author information is available at the end of the article
}

(c) The Author(s). 2020 Open Access This article is licensed under a Creative Commons Attribution 4.0 International License, which permits use, sharing, adaptation, distribution and reproduction in any medium or format, as long as you give appropriate credit to the original author(s) and the source, provide a link to the Creative Commons licence, and indicate if changes were made. The images or other third party material in this article are included in the article's Creative Commons licence, unless indicated otherwise in a credit line to the material. If material is not included in the article's Creative Commons licence and your intended use is not permitted by statutory regulation or exceeds the permitted use, you will need to obtain permission directly from the copyright holder. To view a copy of this licence, visit http://creativecommons.org/licenses/by/4.0/. The Creative Commons Public Domain Dedication waiver (http://creativecommons.org/publicdomain/zero/1.0/) applies to the data made available in this article, unless otherwise stated in a credit line to the data. 


\begin{abstract}
(Continued from previous page)
Conclusions: The findings suggest very low to moderate certainty of evidence that exercise therapy may result in little or no important difference in pain or disability, compared with other interventions, in adult patients with acute low back pain. A limitation of this systematic review is that some included reviews were of low quality. When implementing findings of this systematic review in clinical practice, patients' preferences and the clinician's expertise also should be considered, to determine if and when exercise therapy should be the intervention of
\end{abstract} choice.

Systematic review registration: PROSPERO: CRD46146, available at: https://www.crd.york.ac.uk/PROSPERO/display_ record.php?RecordID $=46146$.

Keywords: Exercise therapy, Acute low back pain, Systematic review, Evidence-based, GRADE

\section{Background}

Low back pain (LBP) affects approximately $70 \%$ of the adult population in the western world, at some point in their lives $[1,2]$. The economic burden for society is high $[1,3]$, and LBP is ranked as one of the three most burdensome conditions in terms of years lived with disability [4]. In the acute phase of LBP, lasting up to 6 weeks, many individuals suffer considerable pain and disability. The prognosis for acute LBP is favorable. Most symptoms resolve within 6 weeks in 70-80\% of affected patients, regardless of intervention or no intervention at all [5-8]. A small proportion, about 5\%, suffer persistent or recurrent pain leading to prolonged disability, which may further abate recovery [6]. Many patients with acute LBP are managed in primary care settings and may visit a physiotherapist [3]. Physiotherapists offer many interventions for this patient group, of which one of the most widely used is exercise therapy [9-11]. This practice is concordant with clinical guidelines for the management of low back pain in primary care [12].

International, evidence-based, clinical practice guidelines exist that can aid the physiotherapist in choosing appropriate interventions for patients with acute LBP [13-15]. However, recommendations in various guidelines, produced in different countries, differ and are not always consistent with results from systematic reviews [13, 16-18]. This may seem odd since systematic reviews of randomized controlled trials (RCTs) provide top level evidence and should be the fundament for any high-quality evidence-based guideline [19]. However, in the development of clinical guidelines, evidence for effectiveness and safety of interventions are only two aspects that are considered. Other aspects such as costs, feasibility, patient preferences, and availability are usually also considered when translating the evidence into recommendations for clinical practice. A mix of populations (acute, sub-acute, and chronic LBP populations) and different definitions of duration are other factors that may explain why recommendations vary $[13,14,16]$. We know that intervention effects differ depending on whether the pain is acute, subacute, or chronic $[17,18,20]$, and that acute pain differs from chronic pain [21].

The most uniform recommendation in international guidelines is that "first line care" for patients with acute LBP should be to give reassurance, advice to stay active in daily life, and, if necessary, pain medication [12, 13]. Exercise therapy, spinal manipulative therapy, mobilization, and acupuncture are other interventions recommended in some, but not all, clinical practice guidelines $[12,13,16]$ and typically if first line care did not lead to improvement of symptoms. For these interventions, there are discrepancies regarding when, how, and whether they should be used in acute LBP $[12,13,15,22]$.

Within the umbrella term exercise therapy, several types of exercise therapy exist. From a physiotherapist perspective, the following types can be distinguished: McKenzie therapy, stabilization exercises (also called motor control exercise), strengthening (or resistance) exercises, stretching exercises, and aerobic exercises. These different types of exercise therapy differ in one important aspect; the hypothesized underpinning effect mechanism. Some have a rather solid theory (physiology), for example aerobic exercise, while others have a more conceptualized theory, for example McKenzie therapy, influenced by the persons that introduced that particular type of exercise therapy. Still, all fit within the umbrella term exercise therapy.

Despite guidelines recommending different interventions, several systematic reviews show that interventions for patients with acute LBP rarely yield clinically relevant effects compared with placebo treatment $[17,23,24]$. This seems to be the case not only concerning physiotherapeutic interventions but for pharmacological treatment as well [25]. Exercise therapy, frequently used in clinical physiotherapy practice [9-11], is no exception from this uncertainty of clinically relevant effect $[7,17,26,27]$. Many systematic reviews conclude that exercise therapy is effective for patient with acute LBP, but that the evidence is 
inconclusive [20, 28]. Although systematic reviews may be well conducted, the often low methodological quality of many of the included studies reduces the confidence we may have in conclusions regarding exercise therapy and its clinically relevant effects [14].

The limitations and issues described imply a need to summarize and synthesize the findings from existing systematic reviews on exercise therapy, and to assess the overall certainty of evidence for effect of this common physiotherapeutic intervention for acute LBP. To the best of our knowledge, no systematic review of systematic reviews on this topic has been published. The aim of this systematic review of systematic reviews was to assess the overall certainty of evidence for the effects of exercise therapy provided by physiotherapists in comparison with other interventions, on pain, disability, recurrence, and adverse effects in adult patients with acute LBP.

\section{Methods}

\section{Protocol and registration}

We conducted this systematic review of systematic reviews according to a protocol registered in PROSPERO (CRD46146), available at: https://www.crd.york.ac.uk/ PROSPERO/display_record.php?RecordID $=46146$. The protocol was not published in any peer-reviewed journal. The development of the protocol was guided by the Preferred Reporting Items for Systematic Review and MetaAnalysis Protocols (PRISMA-P) 2015 statement [29]. Conduct and reporting followed Smith et al.'s [30] methodological recommendations for conducting a systematic review of systematic reviews of healthcare interventions, the Cochrane Collaboration's recommendations for conducting an overview of systematic reviews [31], Lunny et al.'s [32, 33] comprehensive publications with recommendation for conducting an overview, and the PRISMA statement [34] (Additional file 1).

\section{Eligibility criteria}

Exercise therapy is defined as "a regimen or plan of physical activities designed and prescribed for specific therapeutic goals, with the purpose to restore normal musculoskeletal function or to reduce pain caused by diseases or injuries" [35]. In this systematic review, interventions were classified as exercise therapy if they could be carried out in physiotherapy and when no other intervention dominated the intervention. For example, the concept of McKenzie therapy involves repeated exercises following a directional preference, but also sometimes spinal mobilization or manipulation [36].

Inclusion criteria and a priori determined definitions for clinical relevance are presented in Table 1.

Excluded populations were patients with acute LBP related to pregnancy, infection, malignity, metastasis, osteoporosis, rheumatic arthritis, fracture, inflammatory process, or radiculopathy (neurologic signs).

\section{Search methods \\ Search strategy}

We designed a comprehensive search strategy with support from a medical librarian. We took guidance from earlier published search strategies in Cochrane Reviews regarding low back pain and exercise therapy, to reach an optimal strategy. We used a wide search strategy to avoid missing relevant systematic reviews not indexed correctly in the databases. Precision of search, calculated as eligible SRs/total records, and number needed to read

Table 1 Inclusion criteria for the current systematic review, including cut-offs for clinical relevance

\begin{tabular}{|c|c|}
\hline Criteria & Description \\
\hline Study design & Systematic review of RCTs. A review was considered systematic if the review authors had identified it as such. \\
\hline Population & $\begin{array}{l}\text { Adult (18-65 years) patients with non-specific acute LBP (onset to } 6 \text { weeks). If the systematic review contained pri- } \\
\text { mary studies on other populations, e.g., adolescents, at least } 70 \% \text { of the included studies had to be on adult popula- } \\
\text { tions. Findings for populations with acute LBP had to be separable from other populations. }\end{array}$ \\
\hline Interventions & Interventions classified as exercise therapy (earlier defined in the background) used by physiotherapists. \\
\hline Comparisons & $\begin{array}{l}\text { Placebo, sham, waiting list, no treatment, usual care, minimal intervention, non-steroid anti-inflammatory drugs } \\
\text { (NSAIDs), analgesics, or other physiotherapeutic interventions. }\end{array}$ \\
\hline Outcomes & Pain intensity (hereafter referred to as pain), disability, recurrence, adverse effects. \\
\hline Length of follow-up & $\begin{array}{l}\text { Post-treatment, short-term (closest to three months), intermediate-term (closest to } 6 \text { months), and long-term (closest } \\
\text { to } 12 \text { months) follow-up. }\end{array}$ \\
\hline $\begin{array}{l}\text { Minimal important difference } \\
(\text { MID) }\end{array}$ & $\begin{array}{l}15 \mathrm{~mm} \text { on the Visual Analogue Scale (VAS) (0-100), } 5 \text { on the Roland Morris Disability Questionnaire (RMDQ) (0-24), } \\
\text { and } 10 \text { for the Oswestry Disability Index (ODI) (0-100) [37]. }\end{array}$ \\
\hline $\begin{array}{l}\text { Clinical relevance for pooled } \\
\text { effect sizes }\end{array}$ & $\begin{array}{l}\text { Small mean difference }(M D)<10 \% \text {; medium MD 10-20\%; large MD }>20 \% \text { of the scale (e.g., }<10 \mathrm{~mm} \text { on a } 100 \mathrm{~mm} \\
\text { VAS). For relative risk: small standardized mean difference }(\mathrm{SMD})<0.4 \text {; medium SMD } 0.41 \text { to } 0.7 \text {; large SMD }>0.7 \\
{[38] \text {. }}\end{array}$ \\
\hline Settings & Primary care physiotherapy or other settings in which the intervention could be practiced, such as home or gym. \\
\hline
\end{tabular}


(NNR), calculated as $1 /$ precision of search, were used to present the search result. No language restrictions were applied. We combined search terms and MESH terms in a search strategy developed for PubMed, and adapted this strategy for the other databases. Search strategies are presented in Additional file 2.

\section{Electronic searches}

We searched PubMed, Cochrane library, CINAHL, PEDro, Web of Science, Open Grey, and PROSPERO for systematic reviews from inception to 1 March 2017. The three latter were explicitly searched for grey literature, including conference abstracts and study protocols. We also searched reference lists published on the website of the McKenzie Institute International [36]. We performed a supplementary search in PubMed in September 2019. As $83 \%$ of the included systematic reviews were indexed in PubMed in the original search, we limited the update search to this database.

\section{Other sources}

We scrutinized the reference lists of included systematic reviews for additional potentially relevant studies. We contacted authors by email if the full text was not available.

\section{Selection of systematic reviews}

Two reviewers ( $\mathrm{MK}$ and $\mathrm{AB}$ or $\mathrm{SB}$ ) independently screened titles and abstracts retrieved from the searches and assessed these for eligibility against the predetermined inclusion criteria (PICOS). We retrieved all titles and abstracts meeting the inclusion criteria in full text. Two independent reviewers $(\mathrm{MK}, \mathrm{SB}$ or $\mathrm{AB})$ read these full text articles to assess eligibility. Disagreements between reviewers were resolved by consensus.

\section{Overlap}

We calculated total overlap (RCTs in included reviews), and overlap for each time point, outcome, and type of exercise therapy, following the formula proposed by Pieper et al. [39]. We present overlap with percentage and corrected covered area (CCA). Interpretation of CCA: $0-5=$ slight overlap, $6-10=$ moderate overlap, $11-15=$ high overlap, and $>15=$ very high overlap.

\section{Assessment of methodological quality of included reviews}

We used A MeaSurement Tool to Assess systematic Reviews (AMSTAR) to assess the methodological quality of the included systematic reviews [40]. AMSTAR has been shown to be a valid and reliable tool to assess methodological quality of systematic reviews [40, 41]. During the process of doing this review, an updated version, AMSTAR 2, was published [42]. Because our assessment was already in progress and the original AMSTAR has more references of reliability and validity in the literature, we chose to continue to use this tool. Two reviewers $(\mathrm{MK}, \mathrm{AB}$ or $\mathrm{SB})$ independently performed this assessment. Before the actual assessment, a pilot test was carried out by five reviewers $(\mathrm{MK}, \mathrm{SB}, \mathrm{AB}, \mathrm{ML}$, and $\mathrm{LN})$ to evaluate interrater reliability for each of the eleven questions. The result showed good interrater reliability (87\% agreement, Fleiss Kappa 0.58), resembling earlier tests [41]. A second pilot test was carried out by $\mathrm{MK}, \mathrm{AB}$, and $\mathrm{SB}$, on another review to examine whether agreement had improved. The second test showed 100\% agreement. Disagreements in the assessments were handled in a consensus dialog after comparing discrepancies between assessors.

\section{Data extraction}

One reviewer $(\mathrm{MK})$ extracted data from the included reviews and another reviewer ( $\mathrm{AB}$ or $\mathrm{SB})$ checked the extraction for accuracy. We extracted the data into a purpose-built data extraction form, adapted from a Cochrane form [43]. We extracted data primarily at the systematic review level, but supplemented this, when necessary, by extracting data at the RCT level. We verified all point estimates at the RCT level. If there were any discrepancies between an RCT and the systematic review in which it was included, we used data from the RCT. We only extracted data from populations with acute LBP. Each conclusion from the reviews and the RCTs on which this conclusion was based was extracted to enable an overall estimate of the evidence.

\section{Data synthesis}

We synthesized the data quantitatively when possible, and otherwise qualitatively. We present the findings from the systematic reviews in summary of findings (SoF) tables for each outcome, type of exercise therapy, and time point. We present continuous data with weighted mean difference (WMD) and 95\% confidence interval (CI), and dichotomous data with risk ratio (RR) and $95 \%$ CI. We used GRADEpro to create the SoF tables [44].

We considered meta-analyses feasible if clinical homogeneity in the comparisons was present, meaning that interventions, comparisons, time points, and outcomes were similar. Clinical homogeneity was assessed by at least two authors (MK and AK or SB) and agreed upon in consensus discussions. We extracted data for the meta-analyses from the original studies. To enable comparison, we rescaled the data for pain to $0-100$ points $(\mathrm{mm})$; e.g., a numeric pain rating score of 3 on a scale from 0 to 10 was rescaled to 30 . The rescaling of the data meant that we could use WMD also for the aggregated effects, which is easier to interpret than the 
standardized mean difference (SMD). Effects were estimated using the inversed variance heterogeneity model, which is a robust estimation method for handling issues of underestimation of the statistical error and overconfident estimates [45]. We defined statistical significance as the $95 \%$ confidence interval not including zero. We used the free meta-analysis software MetaXL 5.3 for the statistical analyses [46].

\section{Assessment of certainty of evidence}

To evaluate certainty in the overall body of evidence, we used the GRADE approach [47]. Certainty of evidence refers to how certain it is that the true effect of an intervention lies within a chosen range or on one side of a specified threshold [48]. In this systematic review, we used either $95 \%$ confidence intervals as the chosen range or the established minimal important difference (MID) as the specified threshold. When available, we used the GRADE and risk of bias assessments made by the authors of the included reviews, for each outcome, comparison, and time point [33]. When not available, we applied GRADE and appraised the potential limitations due to risk of bias, inconsistency, imprecision, and indirectness ourselves, based on the original studies. We did not assess publication bias due to the small number of studies in most comparisons.

\section{Results}

\section{Search results}

The searches retrieved 2602 records. After screening of titles and abstracts, 134 full-text assessments were carried out. We included 24 systematic reviews with a total of 572 RCTs (overlap not accounted for). Numbers needed to read was 103 and the precision of the search was $0.97 \%$. Six of the 24 reviews were Cochrane reviews. Of the 572 RCTs, 25 publications reporting findings from 21 RCTs with a total of 2685 participants examined exercise therapy for acute LBP. Data for a total of 69 comparisons were extracted from the reviews. Eleven RCTs, with a total of 1397 participants, were included in meta-analyses. Overlap was high, $76 \%$, with a corrected covered area of 0.14. The flowchart in Fig. 1 illustrates the selection process. The supplementary search performed in September 2019 did not result in any additional reviews that met the inclusion criteria.

\section{Description of included reviews}

The included reviews were published between 1993 and 2018. Included RCTs in the reviews with data for an acute population were published between 1982 and 2013. Characteristics of the included reviews are presented in Table 2. Excluded reviews are presented in Additional file 3, with reason for exclusion.

\section{Exercise therapy}

Stabilization exercise and McKenzie therapy were two specific types of exercise therapy in the included reviews that were possible to classify and assess separately. When a mix of different types of exercise was used as the intervention, we used the term general exercise therapy. For stabilization exercise (including co-contraction of multifidi and transversus abdominis muscles or facilitation of abdominal and/or lumbar extensor muscles, initially at low levels of contraction with progression), various terms were used in the reviews: stabilization exercises [82], specific stabilization exercises [68], specific spinal stabilization exercises [78], segmental stabilizing exercises [28], or motor control exercises [17, 84]. McKenzie therapy [70], directional preference management [88], directional preference exercise [87], McKenzie approach [81], and McKenzie method [76, 89] were used to describe the concept officially named The McKenzie Method ${ }^{\circledR}$ of Mechanical Diagnosis and Therapy ${ }^{\oplus}$ (2018).

\section{Comparisons}

The following interventions were used as comparator: usual care, advice, educational booklet, general practitioner management, medical management, spinal manipulative therapy, manual therapy, NSAID, activity of daily life, no treatment, bed rest, and sham ultrasound.

\section{Treatment duration and frequency}

Treatment periods for the exercise therapy groups ranged from 3 days to 8 weeks. Frequency ranged from one to three visits per week. Additional home exercise frequency ranged from three times per day to once every hour.

\section{Outcomes}

Different outcomes were reported in the systematic reviews: pain, bothersomeness, function, functional status, disability, recurrence, patient satisfaction, global improvement, time to recovery, mobility, loss of work days, back to work, muscle thickness, sick leave, activity of daily living, and adverse effects.

\section{Outcome measures}

Pain was measured using the Visual Analogue Scale (VAS) or the Numeric Rating Scale (NRS). Disability was measured using the Roland Morris Disability Questionnaire (RMDQ) or the Oswestry Disability Index (ODI). Data were often transformed and presented with MD or SMD, with $95 \%$ CI or standard deviation (SD). Recurrence was measured as frequency or number of patients affected, and transformed, when possible, to risk ratio (RR) with 95\% CI. Otherwise (more frequently in older systematic reviews), outcome measures from original 


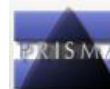

PRISMA 2009 Flow Diagram
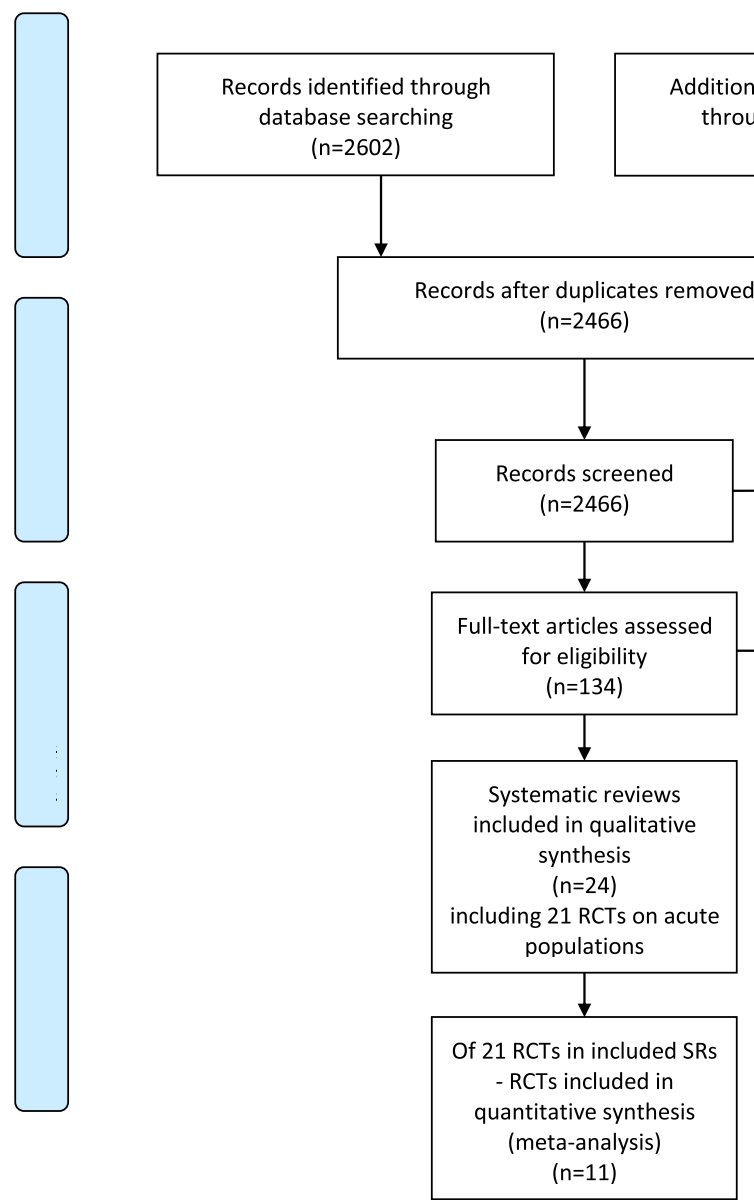

alional records identified hrough other sources $(n=0)$

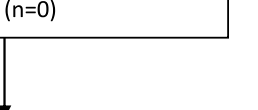

政




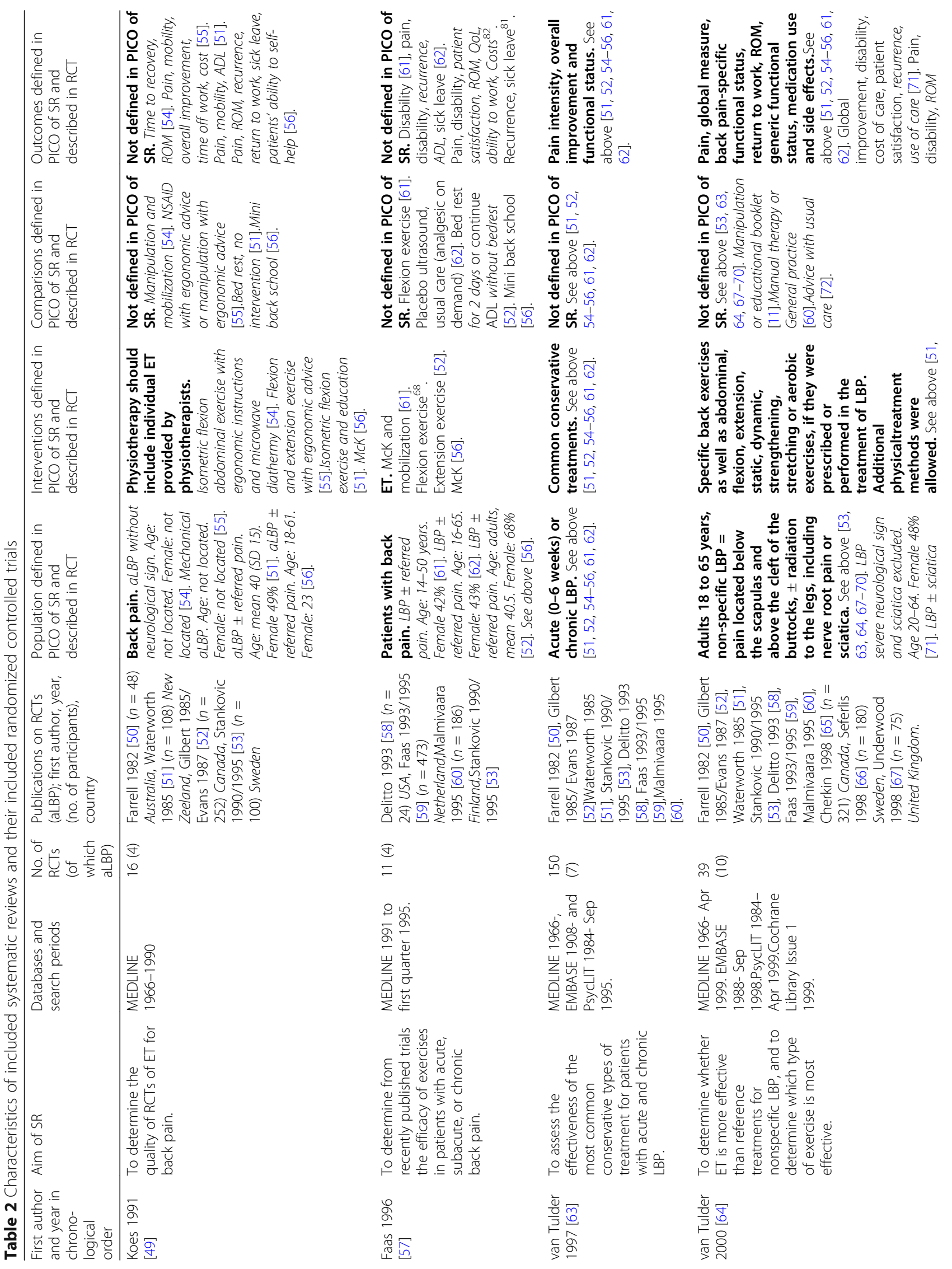




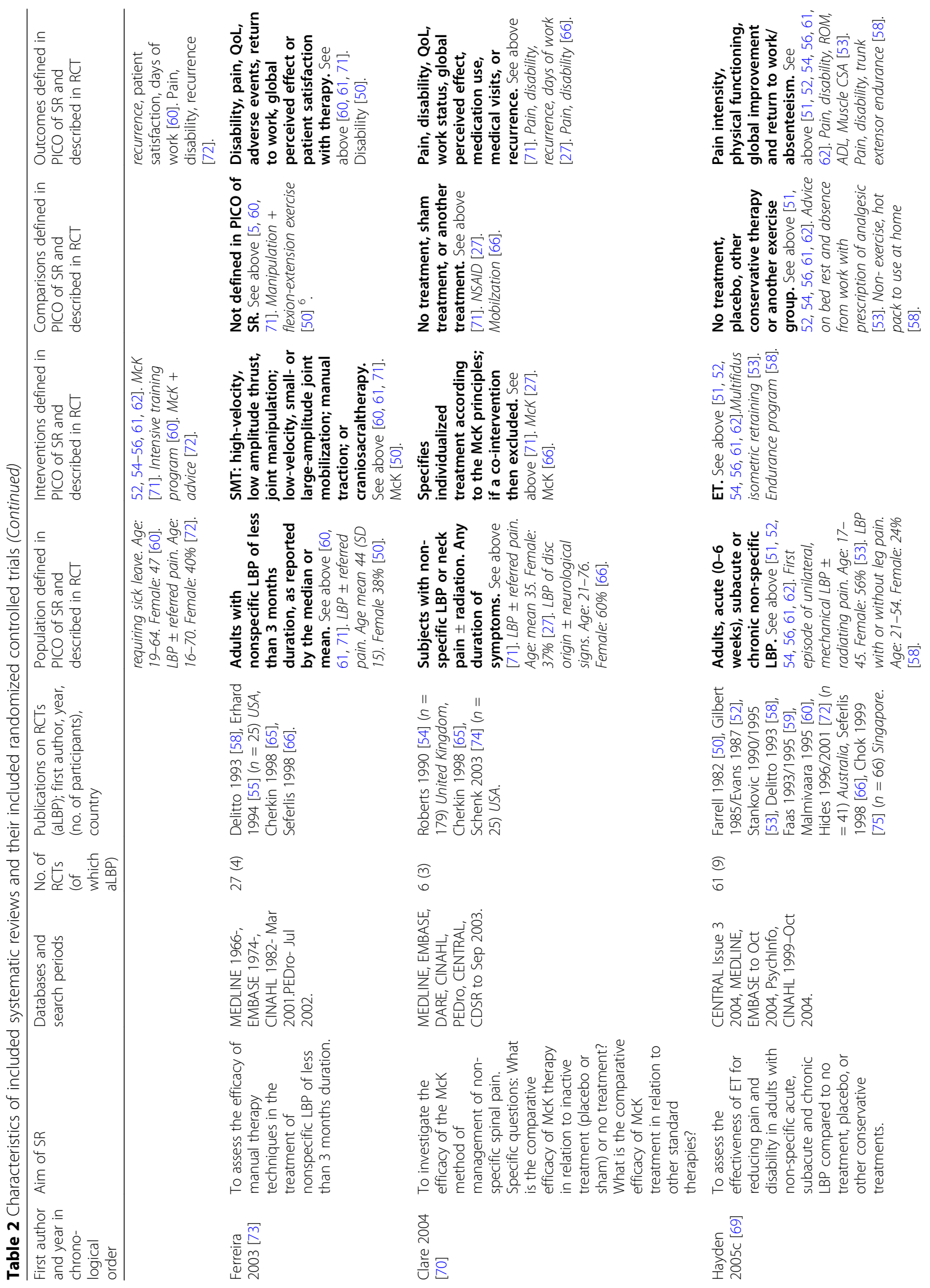




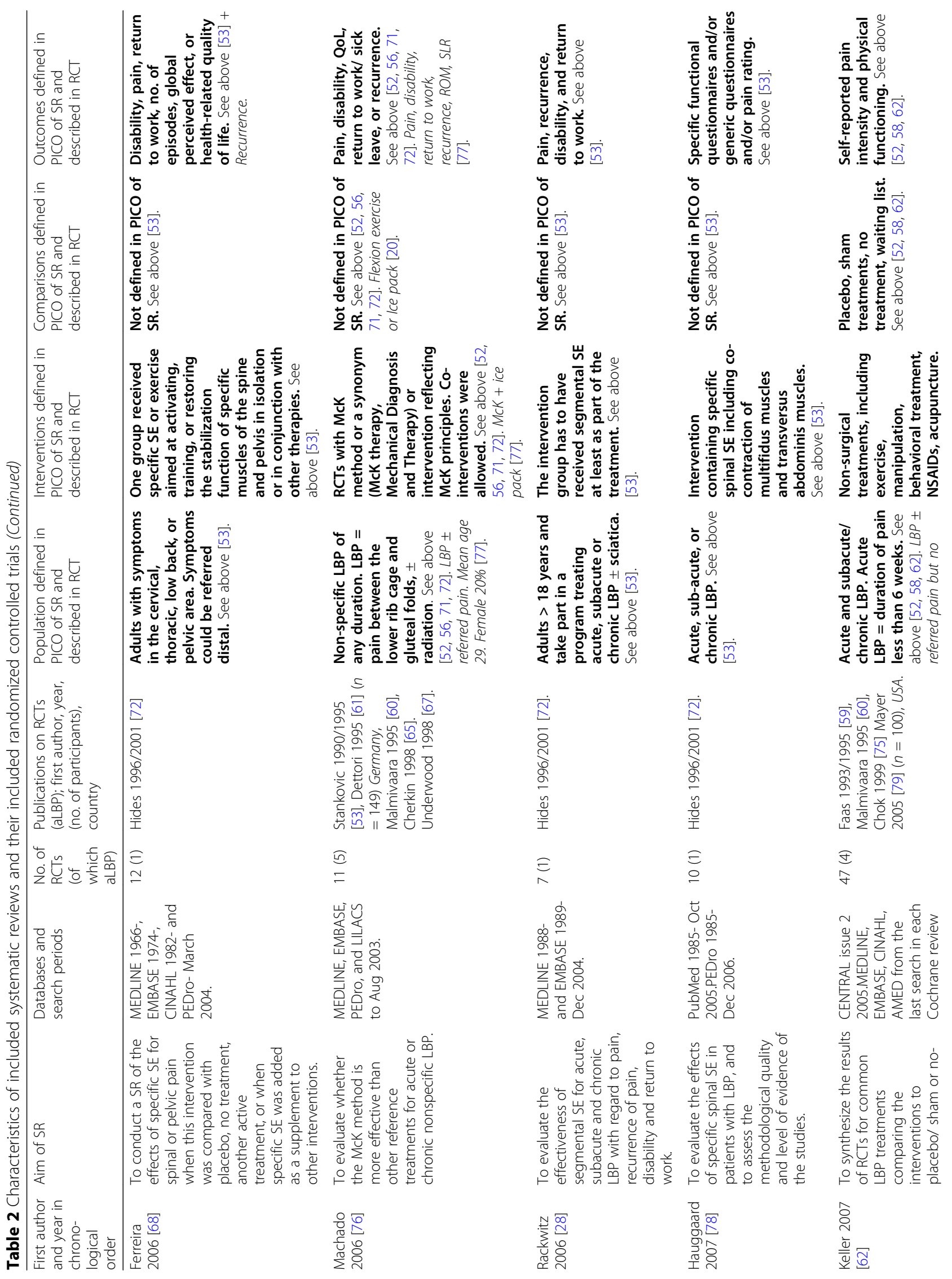




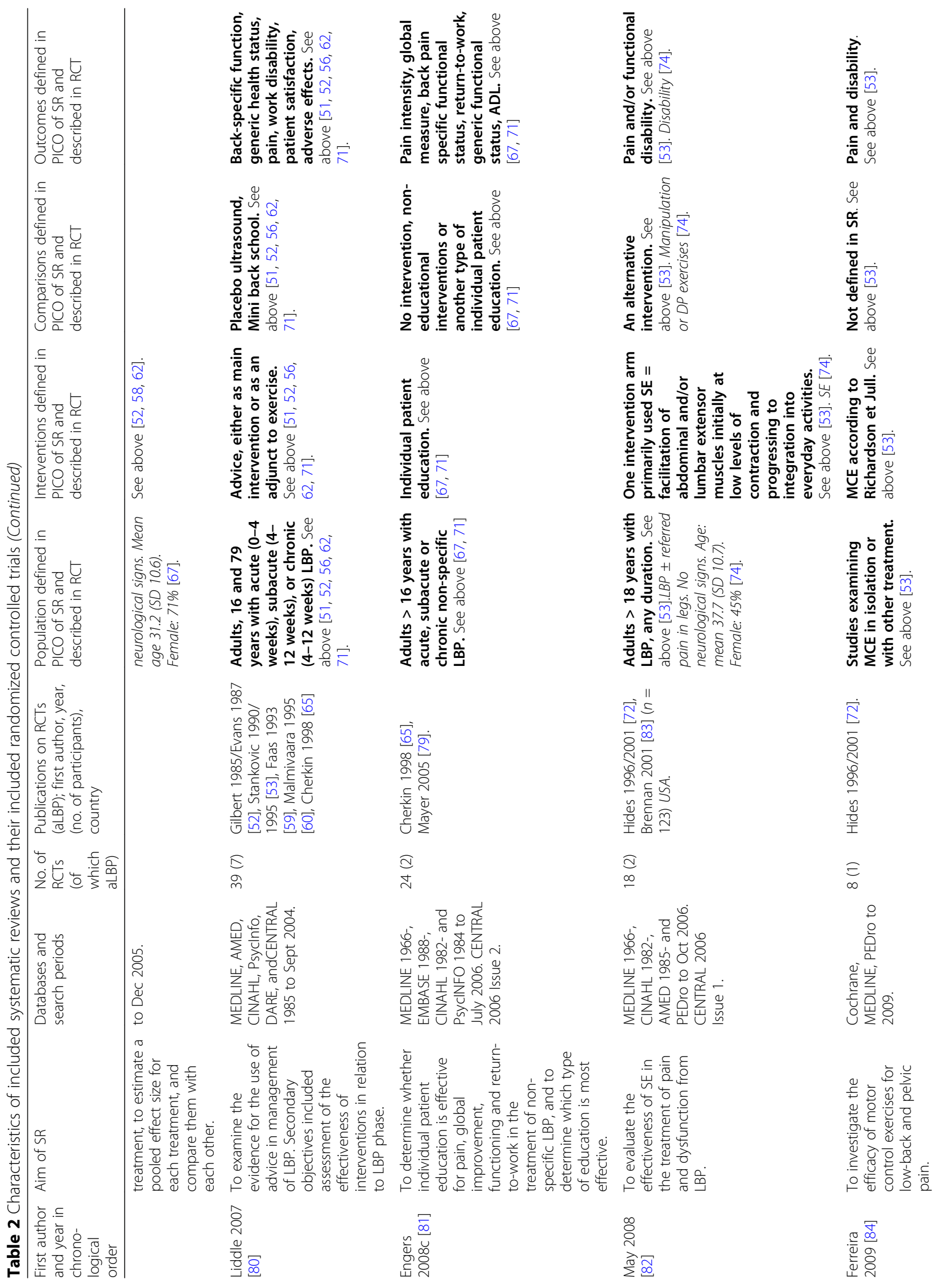




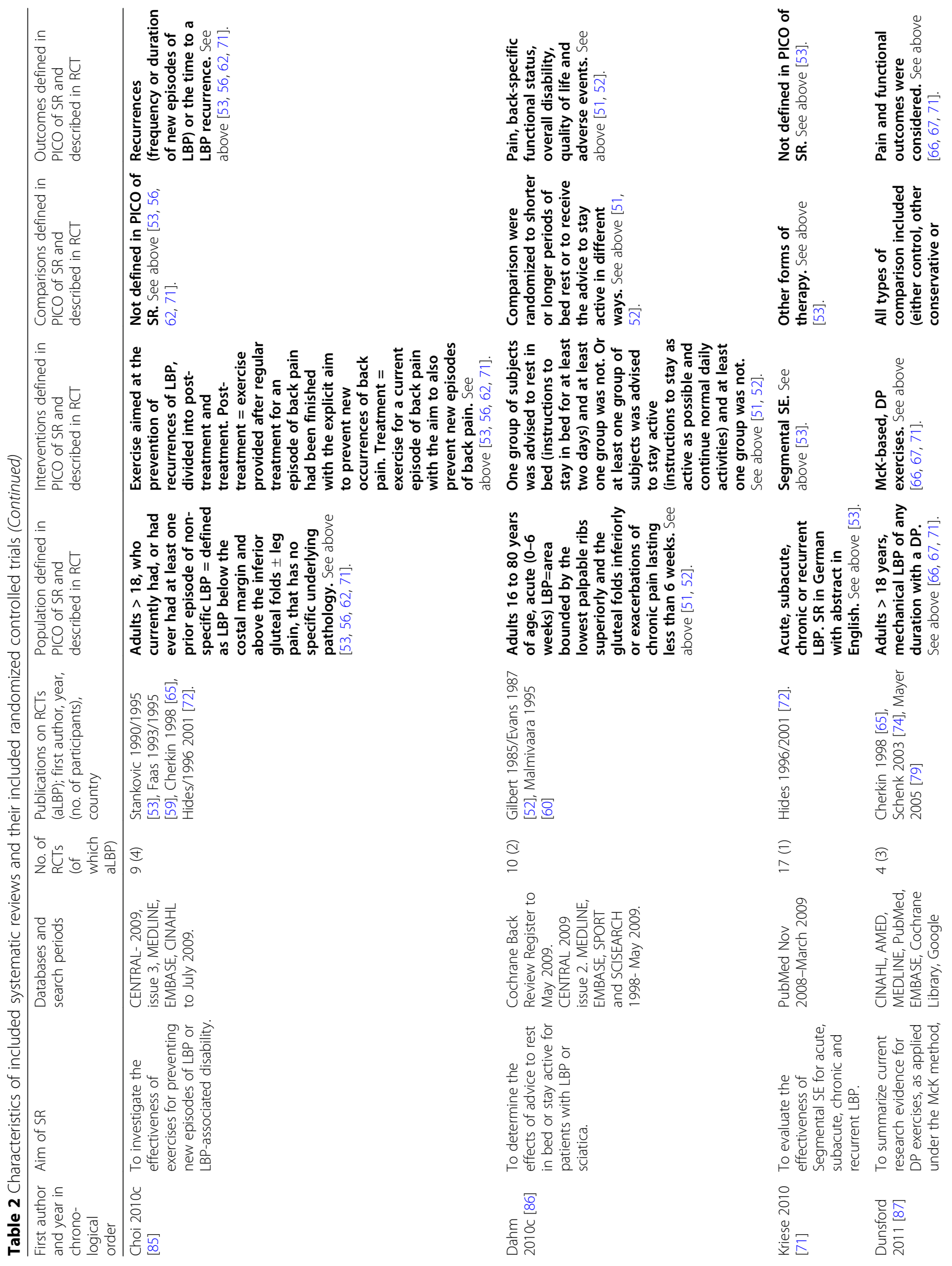




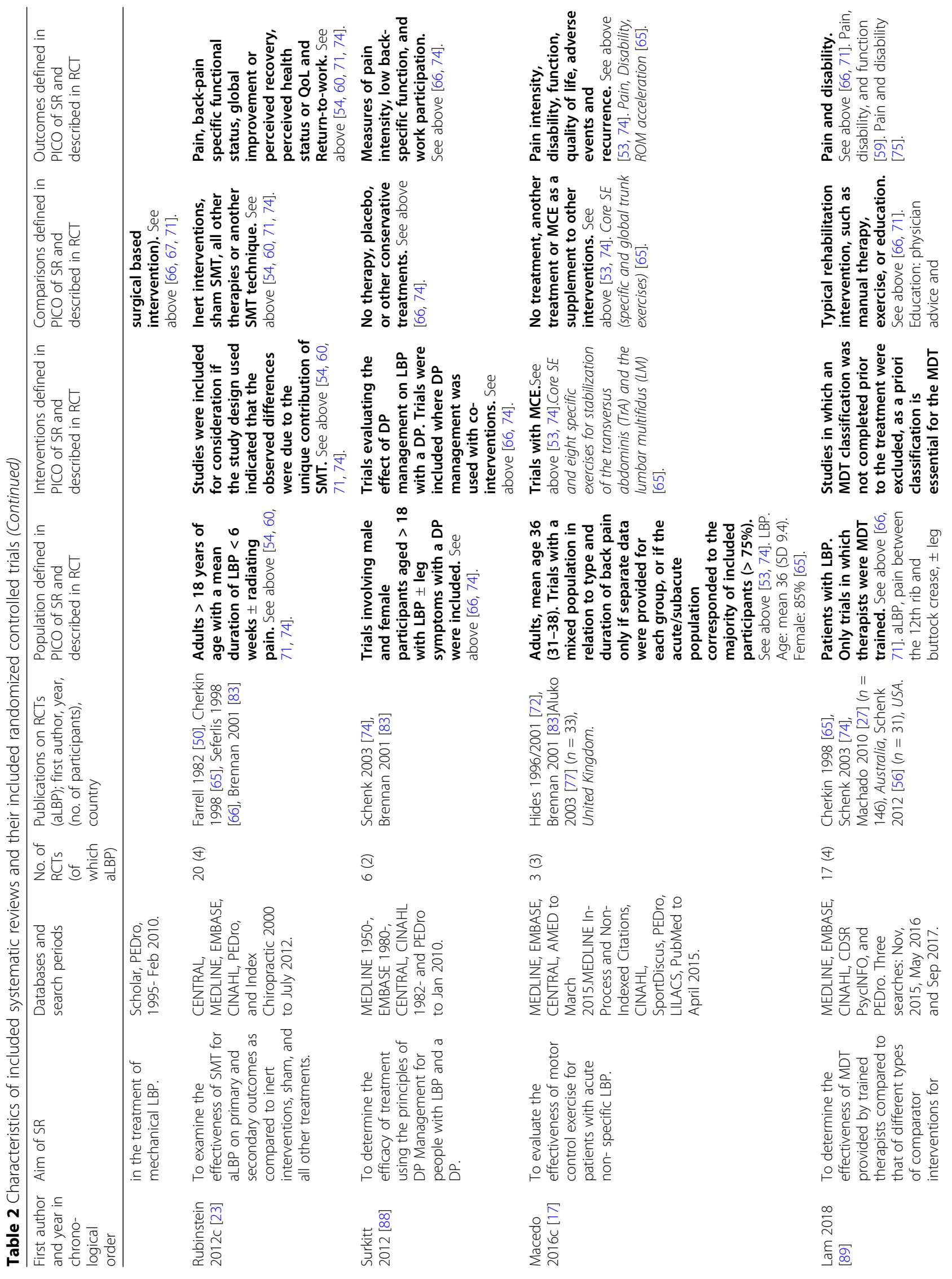




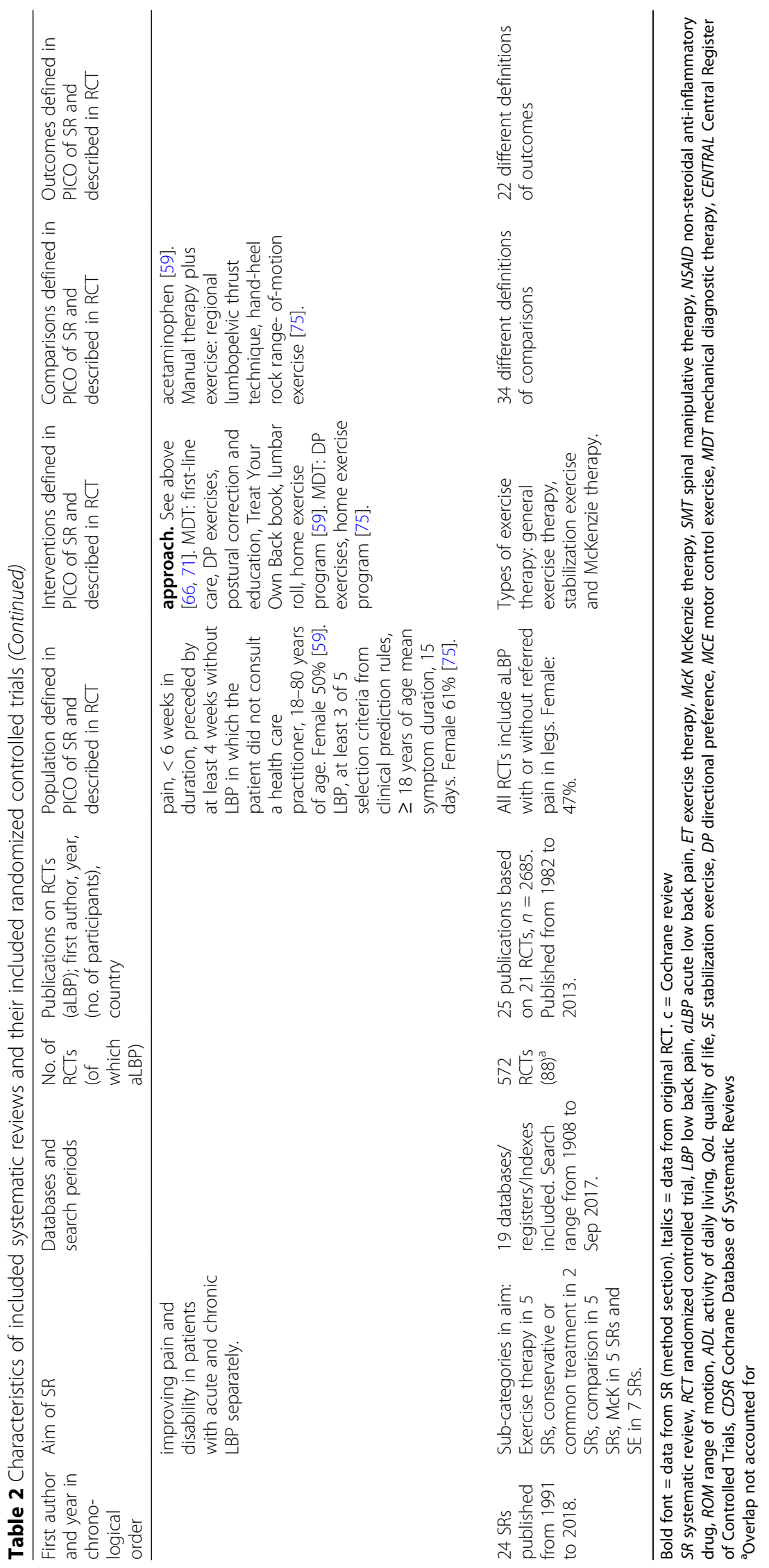


Table 3 AMSTAR quality assessment of included reviews

\begin{tabular}{|c|c|c|c|c|c|c|c|c|c|c|c|c|}
\hline \multicolumn{13}{|l|}{ AMSTAR questions } \\
\hline \multicolumn{13}{|c|}{ 1. Was an 'a priori' design provided? } \\
\hline \multicolumn{13}{|c|}{ 2. Was there duplicate study selection and data extraction? } \\
\hline \multicolumn{13}{|c|}{ 3. Was a comprehensive literature search performed? } \\
\hline \multicolumn{13}{|c|}{$\begin{array}{l}\text { 4. Was the status of publication (i.e. grey literature) used as an inclusion } \\
\text { criterion? }\end{array}$} \\
\hline \multicolumn{13}{|c|}{ 5. Was a list of studies (included and excluded) provided? } \\
\hline \multicolumn{13}{|c|}{ 6. Were the characteristics of the included studies provided? } \\
\hline \multicolumn{13}{|c|}{$\begin{array}{l}\text { 7. Was the scientific quality of the included studies assessed and } \\
\text { documented? }\end{array}$} \\
\hline \multicolumn{13}{|c|}{$\begin{array}{l}\text { 8. Was the scientific quality of the included studies used appropriately in } \\
\text { formulating conclusions? }\end{array}$} \\
\hline \multicolumn{13}{|c|}{$\begin{array}{l}\text { 9. Were the methods used to combine the findings of studies } \\
\text { appropriate? }\end{array}$} \\
\hline \multicolumn{13}{|c|}{ 10. Was the likelihood of publication bias assessed? } \\
\hline \multicolumn{13}{|c|}{ 11. Was the conflict of interest included? } \\
\hline Review/Question & 1 & 2 & 3 & 4 & 5 & 6 & 7 & 8 & 9 & 10 & 11 & Sum \\
\hline oes 1991 & - & - & - & - & Y & - & Y & Y & Y & - & - & $4 / 11$ \\
\hline aas 1996 & - & - & - & - & Y & - & Y & Y & - & - & - & $3 / 11$ \\
\hline anTulder 1 & - & - & - & - & - & Y & Y & Y & Y & - & - & $4 / 11$ \\
\hline /anTulder 2000 & Y & Y & Y & - & Y & Y & Y & Y & Y & - & - & $8 / 11$ \\
\hline Eerreira 2003 & - & - & Y & - & Y & - & Y & Y & Y & - & - & $5 / 11$ \\
\hline Iare 2004 & - & Y & Y & Y & Y & - & - & Y & Y & - & - & $6 / 11$ \\
\hline Hayden 2005 & $\mathrm{Y}$ & $\mathrm{Y}$ & $Y$ & $\mathrm{Y}$ & $\mathrm{Y}$ & $\mathrm{Y}$ & $Y$ & $Y$ & $Y$ & $\mathrm{Y}$ & - & $10 / 11$ \\
\hline 2006 & - & - & Y & Y & - & - & - & Y & Y & - & - & $4 / 11$ \\
\hline 150 onda 2006 & - & Y & Y & - & Y & Y & Y & Y & Y & - & - & $7 / 11$ \\
\hline Rackwitz 2006 & - & Y & Y & - & Y & Y & Y & Y & Y & - & - & $7 / 11$ \\
\hline 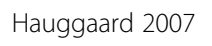 & - & - & Y & - & - & Y & Y & Y & - & - & - & $4 / 11$ \\
\hline Keller 2007 & - & - & - & - & $Y$ & - & Y & Y & Y & - & - & $4 / 11$ \\
\hline Liddle 2007 & - & Y & Y & Y & Y & Y & Y & Y & Y & - & - & $8 / 11$ \\
\hline Engers 2008 & $Y$ & $Y$ & $Y$ & - & $\mathrm{Y}$ & $\mathrm{Y}$ & $Y$ & $Y$ & $Y$ & - & - & $8 / 11$ \\
\hline May 2008 & - & - & Y & - & - & Y & - & Y & Y & - & - & $4 / 11$ \\
\hline 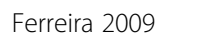 & - & - & - & - & - & Y & Y & Y & - & - & - & $3 / 11$ \\
\hline Choi 2010 & $\mathrm{Y}$ & $Y$ & $Y$ & - & $\mathrm{Y}$ & $\mathrm{Y}$ & $Y$ & $Y$ & $\mathrm{Y}$ & $\mathrm{Y}$ & - & $9 / 11$ \\
\hline Dahm 2010 & $\mathrm{Y}$ & $\mathrm{Y}$ & $\mathrm{Y}$ & $\mathrm{Y}$ & $\mathrm{Y}$ & $\mathrm{Y}$ & $Y$ & $Y$ & $Y$ & $\mathrm{Y}$ & - & $10 / 11$ \\
\hline Kriese 2010 & - & - & - & - & - & Y & - & Y & - & - & - & $2 / 11$ \\
\hline Dunsford 2011 & - & - & - & - & - & Y & Y & Y & Y & - & - & $4 / 11$ \\
\hline Rubinstein 2012 & $\mathrm{Y}$ & $Y$ & $Y$ & $\mathrm{Y}$ & $\mathrm{Y}$ & $\mathrm{Y}$ & $\mathrm{Y}$ & $\mathrm{Y}$ & $Y$ & $\mathrm{Y}$ & $\mathrm{Y}$ & $11 / 11$ \\
\hline Surkitt 2012 & Y & Y & Y & Y & - & Y & Y & Y & Y & - & - & $8 / 11$ \\
\hline Macedo 2016 & $\mathrm{Y}$ & $\mathbf{Y}$ & $Y$ & $\mathrm{Y}$ & $\mathrm{Y}$ & $\mathrm{Y}$ & $Y$ & $Y$ & $\mathrm{Y}$ & $\mathrm{Y}$ & $\mathrm{Y}$ & $11 / 11$ \\
\hline Lam 2018 & - & Y & Y & Y & - & Y & Y & Y & Y & - & - & $7 / 11$ \\
\hline
\end{tabular}

$\mathrm{Y}=\mathrm{Yes} ;-=$ no or cannot answer; bold text $=$ Cochrane review

Quality assessment of the included RCTs, as assessed by the authors of the included reviews

Four RCTs [27, 59, 65, 77] were consistently assessed as being of high quality, two RCTs of moderate quality [54,
61], and six RCTs [50, 51, 55, 56, 58, 75] of low quality. The assessment of the remaining nine RCTs [52, 53, 60, $66,67,72,74,79,83$ ] varied between low and high quality. The main limitations of the RCTs were small sample sizes and lack of blinding of participants, intervention providers, and outcome assessors.

\section{Review conclusions for acute populations}

Twenty-one of the 24 included reviews concluded that there was no difference in effects and three reviews made no conclusive statement about the difference in effect between exercise therapy and any comparator for the acute population. Three reviews concluded that there were positive effects of exercise therapy, but only for the outcome recurrence at long-term follow-up. However, this was based on the same, single RCT [72]. Of the RCTs included in the reviews, four showed results in favor of exercise therapy for some outcomes, 14 resulted in no difference, and three RCTs showed results in favor of the comparator.

\section{Outcomes}

Findings are summarized below and presented in detail in SoF tables 4-12 (Additional file 4).

\section{Pain}

General exercise therapy Twelve reviews [17, 23, 49, $57,62-64,69,73,76,80,86]$, including eight RCTs $[50-52,59,61,66,75,77]$ of low to high quality, addressed effects of general exercise therapy on pain. Overlap for the various time points ranged from 75 to $100 \%$, with corrected covered areas of $0.25-0.70$.

We were able to pool data for one comparison. Metaanalysis of four RCTs [51, 59, 66, 75] comparing general exercise therapy with usual care showed no significant difference in post-treatment effects on pain (Fig. 2).

No important difference in effects of general exercise therapy on pain was reported for any comparison or time point (SoF table 4). Evidence ranging from very low to moderate certainty suggests that general exercise therapy probably results in little or no important difference in pain, at any time point, when compared with any of the investigated control interventions.

Stabilization exercise Seven reviews [17, 28, 68, 71, 78, $82,84]$, including three RCTs [72, 77, 83] of low to high quality, addressed effects of stabilization exercise on pain. Overlap was $67 \%$ with a corrected covered area of 0.39 . No important differences in effects of stabilization exercise on post-treatment or short-term pain were reported (SoF table 5). Intermediate- or long-term effects were not reported. Evidence ranging from low to moderate certainty suggests no important difference in pain at post-treatment and short-term, when comparing 


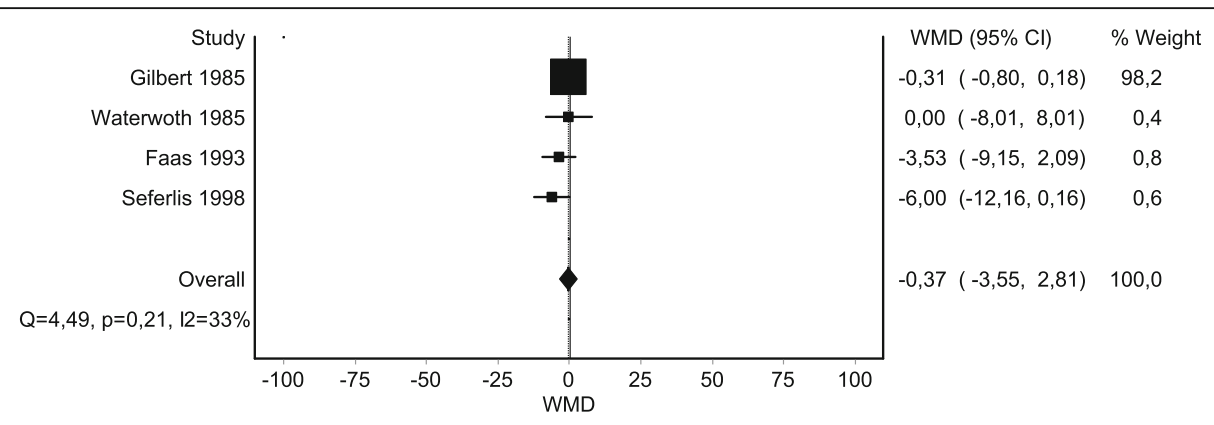

Fig. 2 Post-treatment effects on pain of general exercise therapy versus usual care

stabilization exercise with other exercise therapies. The evidence is very uncertain whether stabilization exercise plus medical management reduces post-treatment pain when compared with medical management alone.

McKenzie therapy Thirteen reviews [23, 49, 62-64, 69, $70,76,81,86-89]$, including seven RCTs [27, 53, 60, 65, $67,74,79]$ of low or high quality, addressed effects of McKenzie therapy on pain. Overlap was $75 \%$ with corrected covered areas of $0.24-0.45$.

We were able to pool data for four comparisons. No significant difference was seen in post-treatment or short-term pain when McKenzie was compared with usual care (Fig. 3a-b) or for McKenzie therapy vs. spinal manipulative therapy post treatment (Fig. 3d). A significant difference was seen between McKenzie therapy and an educational booklet: total MD - 11.30 (95\% CI - 18.15 to -4.45 ) (Fig. 3c). However, the effect did not exceed the MID of $15 \mathrm{~mm}$ on the VAS. Findings for other comparisons are presented in SoF table 6.

At intermediate and long term, no important difference in effects of McKenzie therapy on pain was reported compared with usual care [69], educational booklet [69], spinal manipulative therapy [65], or NSAID [54]. Evidence ranging from very low to moderate certainty suggests no important difference in pain at any time point, when comparing McKenzie therapy with any of the control interventions.

\section{Disability}

General exercise therapy Nine reviews $[23,49,62-64$, $69,73,80,86]$, including six RCTs [50-52, 59, 66, 75] of predominantly low to moderate quality, addressed effects of general exercise therapy on disability. Overlap was $100 \%$ with corrected covered areas of $0.33-0.40$.

We were able to pool data for three comparisons. Meta-analysis of three RCTs [52, 59, 66] of general exercise therapy versus usual care showed a statistically significant difference in post-treatment effects on disability in favor of usual care: MD 2.62 (95\% CI 0.52 to 4.72 )
(Fig. 4a). However, this effect did not exceed the MID. Meta-analysis of two RCTs $[59,66]$ on short-term effects and of two RCTs $[59,66]$ on long-term effects of disability of general exercise therapy versus usual care showed no significant difference (Fig. 4b, c, SoF table 7).

No important difference in effects of general exercise therapy on post-treatment disability was reported compared with sham ultrasound [69], spinal manipulative therapy [69], hot pack [69], or NSAID [64]. In comparison with sham ultrasound [69], hot-pack [69], bed rest, or usual care [64], no important difference in effects of general exercise therapy on short-term disability was reported. None of the included reviews reported intermediate-term effects. In comparison with sham ultrasound [69] or bed rest [64], no important difference in long-term effects of general exercise therapy on disability was reported. Evidence of low to moderate certainty suggests no important difference in disability at any time point, when comparing general exercise therapy and usual care.

Stabilization exercise Seven reviews [17, 28, 68, 71, 78, $82,84]$, including three RCTs [72, 77, 83] of low to high quality, addressed effects of stabilization exercise on disability. Overlap was $67 \%$ with a corrected covered area of 0.39 . No important difference in effects of stabilization exercise in post-treatment, short-term, or long-term disability was reported (SoF table 8). No intermediate-term effects were reported. Evidence of very low to low certainty suggests no important difference in disability at any time point, when stabilization exercise is compared with any of the control interventions examined. The evidence is very uncertain whether stabilization exercise plus medical management reduces post-treatment disability when compared with medical management alone.

McKenzie therapy Seven reviews [23, 69, 70, 76, 8789], including seven RCTs [27, 60, 65, 67, 74, 79, 83] ranging from low to high quality, addressed effects of 


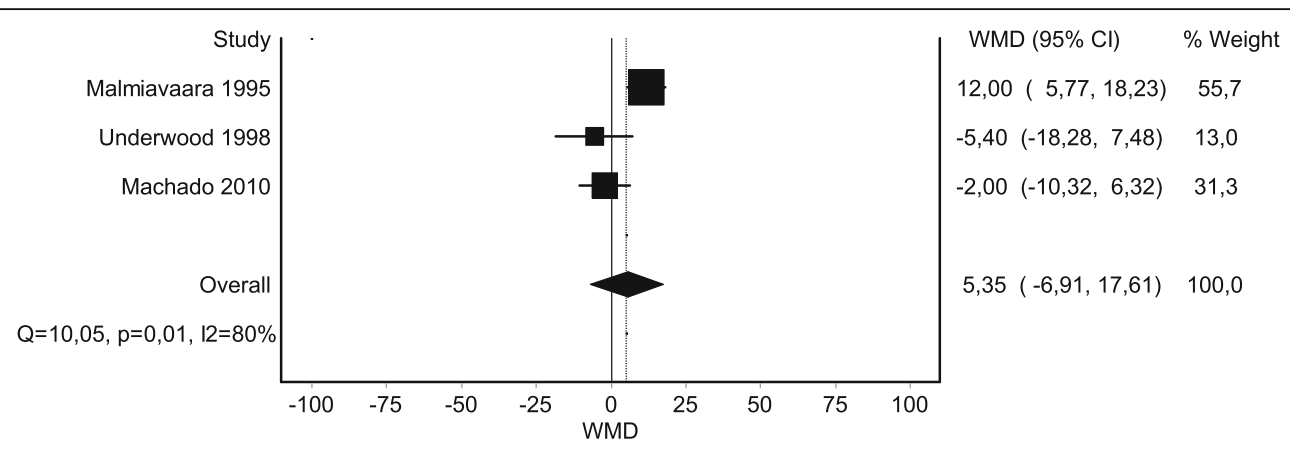

a. McKenzie vs. usual care, post treatment

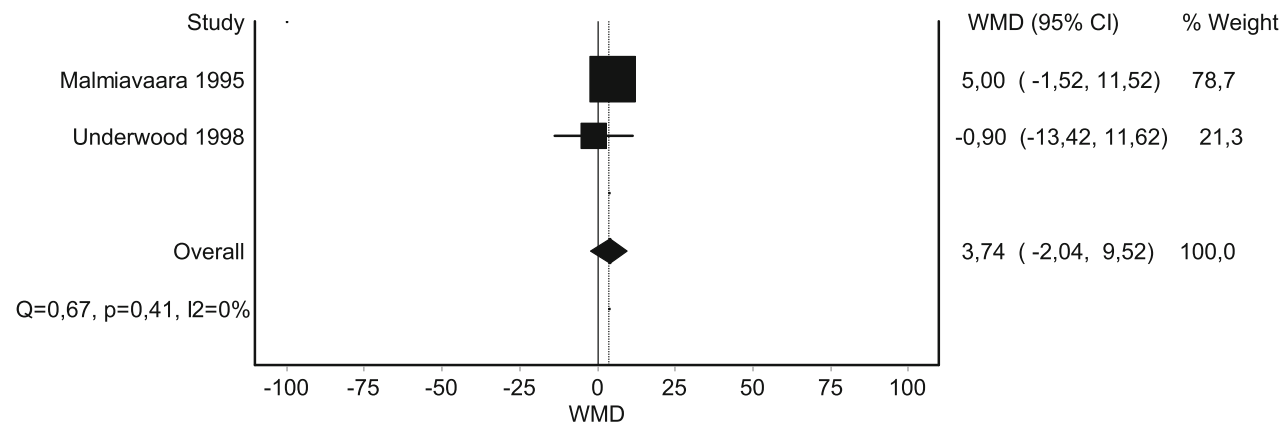

b. McKenzie vs. usual care, short term

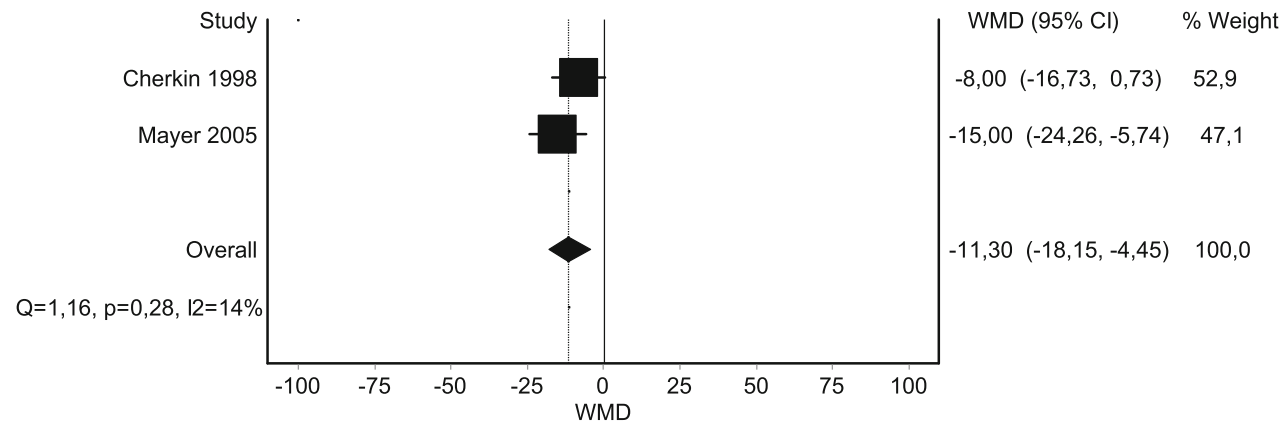

c. McKenzie vs. education booklet, post treatment

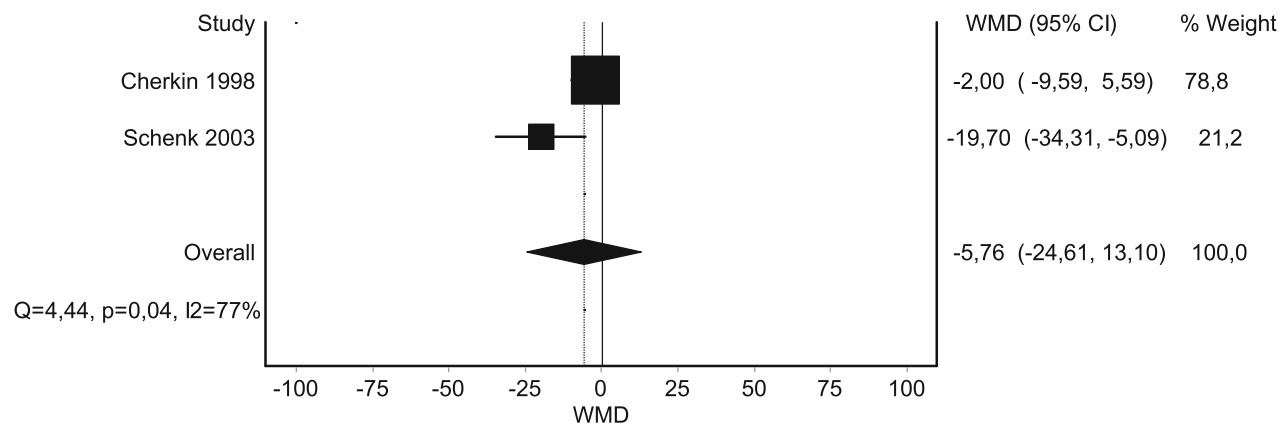

d. McKenzie vs. spinal manipulative therapy, post treatment

Fig. 3 a McKenzie vs. usual care, post treatment. b McKenzie vs. usual care, short term. c McKenzie vs. education booklet, post treatment. d McKenzie vs. spinal manipulative therapy, post treatment 


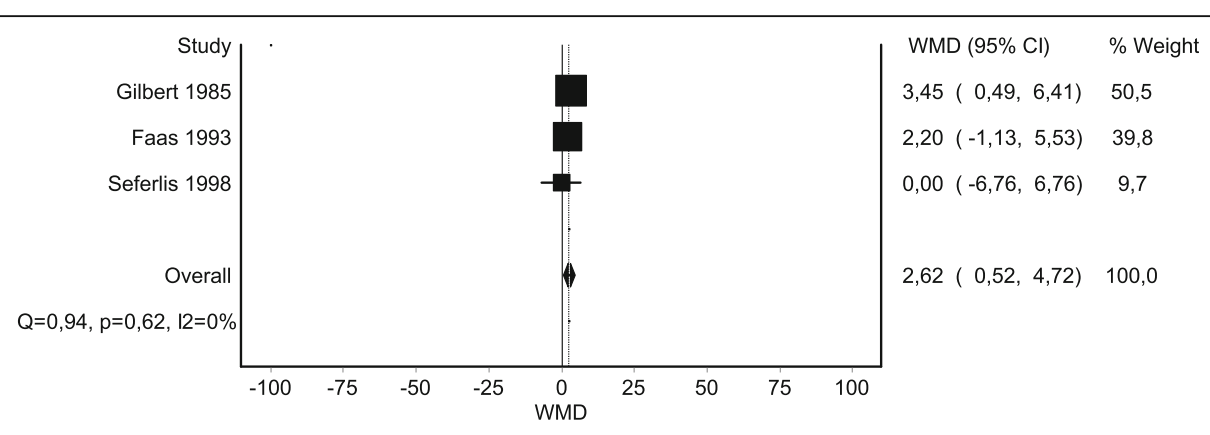

a. General exercise therapy vs. usual care, post treatment

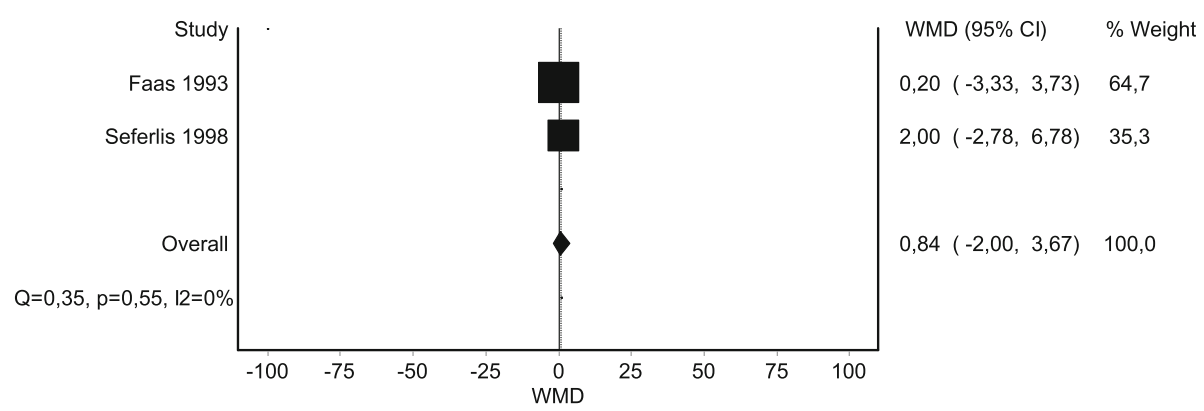

b. General exercise therapy vs. usual care, short term

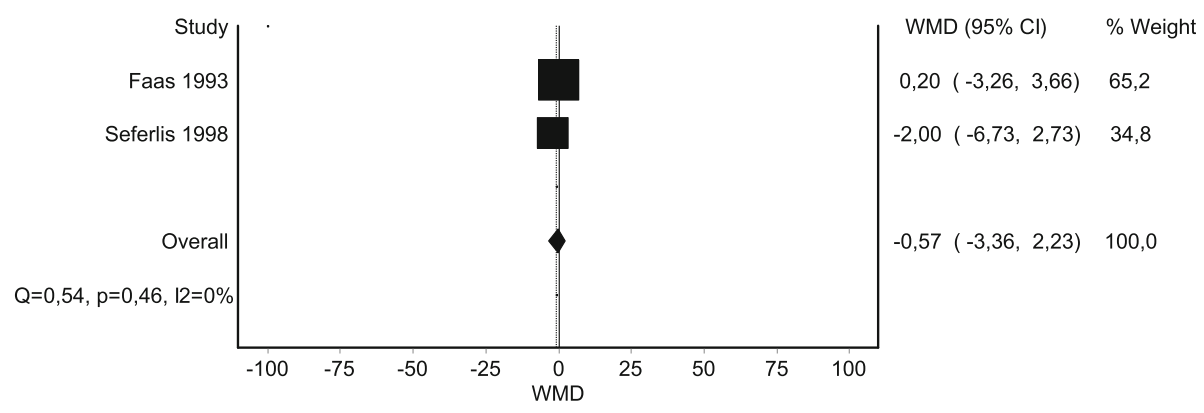

c. General exercise therapy vs. usual care, long term

Fig. 4 a General exercise therapy vs. usual care, post treatment. b General exercise therapy vs. usual care, short term. c General exercise therapy vs. usual care, long term

McKenzie therapy on disability. Overlap was $71-75 \%$ with corrected covered areas of $0.25-0.42$.

We were able to pool data for four comparisons. No important differences were seen in post-treatment or short-term disability when McKenzie therapy was compared with usual care, educational booklet, or spinal manipulative therapy (Fig. 5a-d).

Findings for other comparisons are presented in SoF table 9. In comparison with educational booklet [70] or NSAID [54], no important difference in intermediateterm effects of McKenzie therapy on disability was reported. In comparison with usual care [69], educational booklet [69], spinal manipulative therapy [23], or NSAID [54], no important difference in long-term effects of McKenzie therapy on disability was reported. Evidence of very low to moderate certainty suggests that there is no difference in disability at any time point, between McKenzie therapy and usual care, spinal manipulative therapy, or NSAID. Evidence of moderate certainty suggests that McKenzie therapy likely does not reduce disability, at any time point, when compared with an educational booklet. 


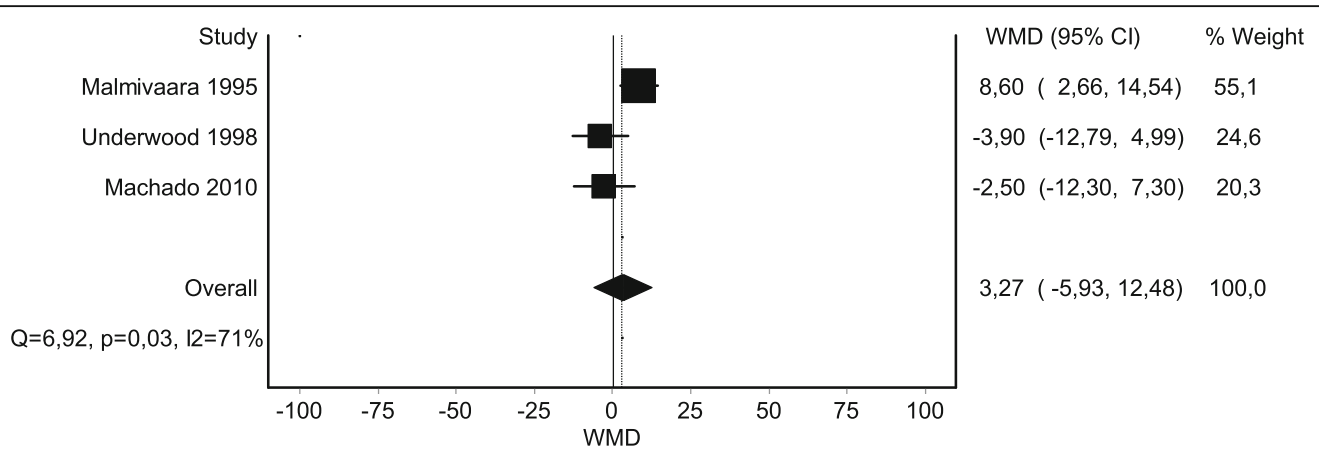

a. McKenzie vs. usual care, post treatment

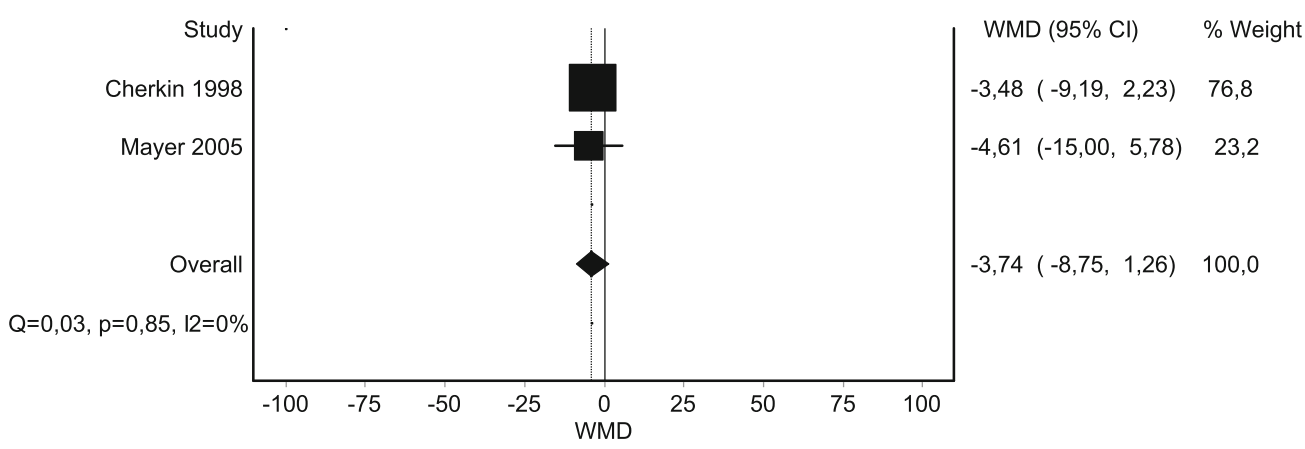

b. McKenzie vs. educational booklet, post treatment

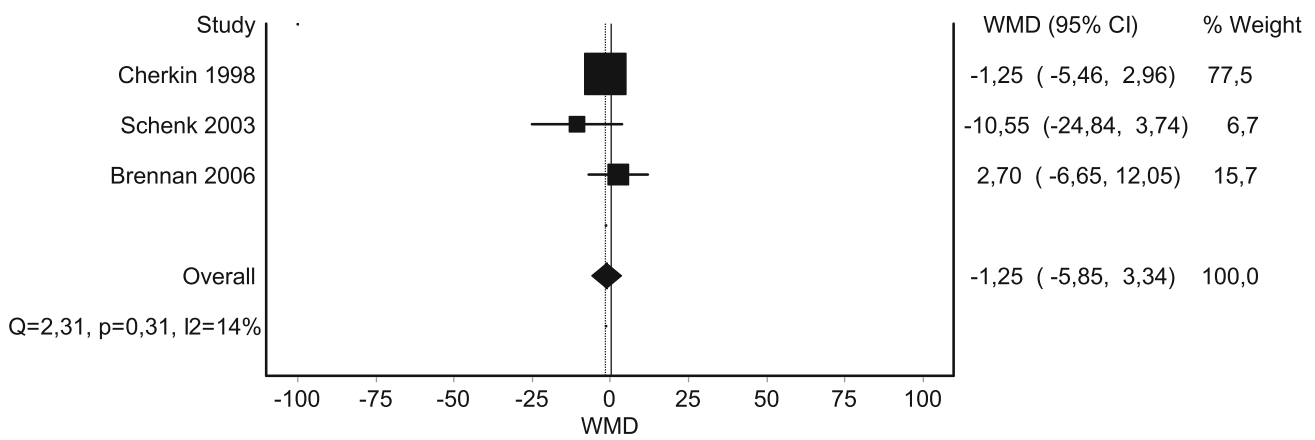

c. McKenzie vs. spinal manipulative therapy, post treatment

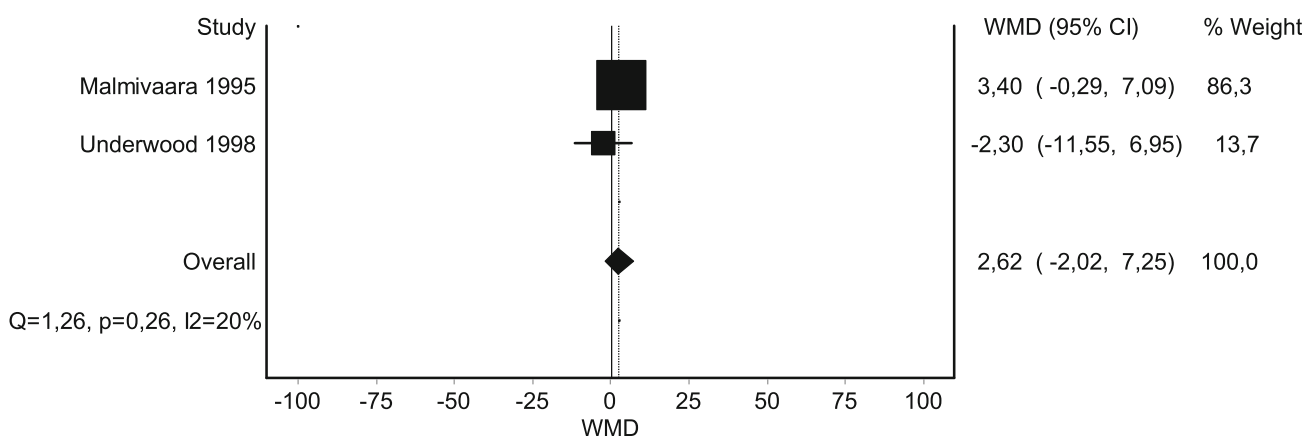

d. McKenzie vs. usual care, short term

Fig. 5 a McKenzie vs. usual care, post treatment. b McKenzie vs. educational booklet, post treatment. c McKenzie vs. spinal manipulative therapy, post treatment. d McKenzie vs. usual care, short term 


\section{Recurrence}

General exercise therapy None of the included reviews addressed post-treatment, short-term, or intermediateterm effects on recurrence. Three reviews [64, 76, 85] addressed long-term effects of general exercise therapy on recurrence. Two of those $[64,85]$ reported results from the same RCT [59]. The third review [76] included one RCT [61] measuring long-term effects on recurrence, but this was not reported in the review. The RCTs were of moderate to high quality. Overlap was $50 \%$ with a corrected covered area of 0.25 .

No significant effects of general exercise therapy on recurrence in comparison with sham ultrasound, usual care, or ice-pack was reported for any time point (SoF table 10). Evidence of moderate certainty suggests that general exercise therapy is not more effective in preventing recurrence than placebo or usual care at long-term. The evidence is very uncertain whether general exercise therapy reduces recurrence when compared with icepack.

Stabilization exercise None of the included reviews reported post-treatment, short-term, or intermediate-term effects of stabilization exercise on recurrence. Eight reviews $[17,28,68,71,78,82,84,85]$ addressed long-term effects of stabilization exercise on recurrence, all based on one RCT [72]. Overlap was $100 \%$ with a corrected covered area of 1.0. Quality assessment of the RCT varied from low to high quality, affecting the level of evidence for stabilization exercise in the reviews, which ranged from very low to moderate evidence. Systematic reviews of low methodological quality tended to overestimate the quality of this RCT. Recurrence was measured as number of persons with recurrence in all eight reviews, and also as frequency of recurrence in one review.

Stabilization exercise plus medical management versus medical management alone resulted in lower relative risk for recurrence, RR 0.36 (95\% CI 0.18 to 0.72 ), while there was no significant difference in recurrence frequency, MD -1.40 (95\% CI -3.16 to 0.36) (SoF table 11). The evidence is very uncertain whether stabilization exercise plus medical management reduces the longterm risk for recurrence when compared with medical management alone.

McKenzie therapy None of the included reviews reported any post-treatment or short-term effects of McKenzie therapy on recurrence. One review [70] addressed intermediate-term effects on recurrence but did not present results that were available in an included RCT [54], assessed as of moderate quality in the review [70]. Three reviews [70, 76, 85] addressed long-term effects on recurrence, in which three RCTs [53, 54, 65], of low to high quality, presented results for McKenzie therapy. Overlap was 50\% with a corrected covered area of 0.25 .

There was no difference in intermediate or long-term recurrence frequency with McKenzie therapy versus NSAID [54], and no long-term effects on recurrence compared with simple back educational interventions (SoF table 12). The evidence is very uncertain whether McKenzie therapy reduces the intermediate or longterm risk for recurrence when compared with simple back education or NSAID.

\section{Adverse effects}

Adverse effects were addressed in ten (42\%) of the reviews $[17,23,28,69,73,80,81,85,86,88]$. No review reported any adverse effects specific to the acute population. Of the included RCTs, two [54, 79] addressed adverse effects, but none of them reported any adverse effects.

\section{Discussion}

The main findings of this systematic review are that moderate-certainty evidence suggests no superior effect of exercise therapy versus any comparator, for any of the examined outcomes, at any time point. Low-certainty evidence suggests that McKenzie therapy may be superior, with a small effect, versus simple educational booklet at post-treatment follow-up for pain; but there were no other differences between McKenzie therapy and other interventions. Very low-certainty evidence suggests that stabilization exercise together with medical management may be superior versus only medical management for recurrence at long-term follow-up, but since the evidence is of very low certainty, we cannot draw any firm conclusions; and there were no other differences between medical management and other interventions.

Adverse effects were rarely addressed in the reviews or in the included RCTs and no adverse effects were reported, indicating a possibility that they were underreported. Mild reactions with increased back pain and muscle soreness were reported in one review [69], but it was unclear in that review whether they were in the acute population or the chronic population.

The body of evidence from 24 systematic reviews consistently shows that exercise therapy in the acute phase of LBP does not yield any clinically important difference compared with any other treatment, for most outcomes and most time points. The most likely explanation for the lack of effect is the generally good prognosis (natural course) of acute LBP. The lack of long-term effect might be explained by factors such as insufficient treatment duration, frequency, or intensity of the exercise protocols. Physiology tells us that the effect of 1 to 8 weeks of exercise may not remain 1 year later, unless exercise is 
maintained [90]. However, relevant post-treatment and short-term effects on pain or disability are also lacking. The conclusion seems justified that the role of exercise therapy in acute LBP is very limited, at best.

The lack of effect of McKenzie therapy has been attributed in previous reviews [70, 76] to the improper use of the method, i.e., without addressing the patient's directional preference. However, the synthesized data in our systematic review do not support any clinically relevant effect, even when this issue has been addressed. A recent review, not included in our systematic review, showed a significant difference in effects on pain and disability between RCTs that adhered to McKenzie core principles and non-adherent RCTs, but no difference between adherent RCTs and other comparators [91].

For stabilization exercise, there is no convincing benefit over other types of exercise therapy.

The most recommended "first line" care (advice to stay active and reassurance of a favorable prognosis) [12, 13] was rarely used as comparison. Instead, a wide variety of interventions, such as spinal manipulative therapy, icepack, hot-pack, NSAID, educational booklets, manual therapy, or medical management with prescribed bed rest, were used as comparison.

Not all included RCTs point in the direction of no effect of exercise therapy. Small RCTs tended to favor exercise therapy, suggesting a potential publication bias. When compared with spinal manipulative therapy, the results often pointed in opposite directions. In contrast, larger trials with low risk of bias pointed in the direction of no effect or no minimal important difference when exercise was compared with less strenuous interventions. The included reviews follow the same pattern; higher quality reviews report no difference, while lower quality reviews suggest a positive effect of exercise therapy. Most lower-quality reviews typically highlight a marginal effect of exercise therapy rather than stating that the effects are not clinically relevant.

The most prominent issues with regard to risk of bias and the resulting uncertainty of the evidence are the small number of RCTs in the included reviews and the small sample size of those RCTs, resulting in a lack of power to detect statistically significant differences. Lack of blinding of patients, intervention providers (which is difficult to do with these interventions), and outcome assessors were potential study limitations that further reduce our confidence in the effects of exercise therapy.

Overlap is an important issue to describe and consider when producing systematic review of systematic reviews [39]. Our systematic review showed a high overlap, which we handled by presenting it with percentage and corrected covered area for each outcome and each comparison. This minimized the risk of bias and enabled us to judge the overall certainty of evidence for the broader term of exercise therapy and the two more specific types, i.e., stabilization exercise and McKenzie therapy.

We are not aware of any other systematic review of systematic reviews addressing exercise therapy for acute LBP. Swinkels et al. [92] addressed the effect of exercise therapy for nonspecific LBP in their overview. That overview included four reviews of which two $[57,69]$ were included in our systematic review. The other two reviews addressed non-acute populations. Swinkels et al. [92] concluded, based on the study by Hayden et al. [69], that exercise therapy is as effective as either no treatment or other non-exercise interventions at short-, intermediate-, and long term follow-up. We do not disagree with that conclusion but conclude, based on the studies included in our systematic review, that exercise therapy does not result in any minimal important difference in effect compared with other interventions. Maher et al. [93] concluded, also based on the study by Hayden et al. [69], that high-quality evidence exists for no difference between exercise therapy versus sham treatment or other conservative treatments. While we agree with their conclusion, our analysis only supports moderate certainty of evidence for this comparison, suggesting that future studies may change our confidence in the estimate of effects.

An updated publication of recommendations in international clinical guidelines showed that exercise therapy is recommended for acute LBP in three of 14 guidelines and that the other 11 guidelines provided inconsistent recommendations on exercise therapy for acute LBP [94]. The Danish guidelines [95] recommend exercise therapy based on low-quality evidence from seven RCTs, including a population with acute LBP (in their definition up to 12 weeks' duration). The authors made the recommendation based on a trend in the results favoring exercise therapy. Five of these RCTs are included in our systematic review. Our findings do not support these recommendations. However, recommendations in guidelines are based on more aspects than solely evidence from systematic reviews. Patients' preferences, clinicians' experiences, costs, availability, and safety are examples of other aspects that are considered and which could explain the discrepancy between recommendations and evidence.

Some of the limitations in this systematic review may have introduced potential biases. The low methodological quality of some of the included reviews and their underlying RCTs contributes to the low certainty of evidence. For most outcomes and time points, the total number of participants was low. The inclusion of the same RCTs in many of the reviews caused a high overlap, and the varying results of review authors' methodological quality assessment of some RCTs is a further cause for concern. Furthermore, our meta-analyses were 
based on aggregate data, which entails a potential for ecological fallacy. A difference in outcomes can be significant in several subgroups, but when combined, this difference may disappear or even reverse; a fallacy known as Simpson's paradox [96]. Our overall GRADE assessment was based on a combination of assessments made by the systematic review authors and ourselves. This combination may entail inconsistency in assessments, as reliability between the assessment made by the authors of the systematic reviews and our research group is unknown.

We have followed available methodological guidance for conducting a systematic review of systematic reviews, but the guidance is evolving and several strategies are available. The choice of strategy will have an impact on the results and conclusion. Overlap could have been minimized by excluding reviews with the same RCTs included. Another possible strategy would have been to exclude reviews of lower methodological quality. However, then we would not have obtained a complete picture of the overall certainty of the evidence from all available reviews.

\section{Implications for practice}

LBP is among the most common reasons for which patients consult a physiotherapist or general practitioner in primary care [97]. It is important to provide accurate, timely, and effective management for this condition. The findings of this systematic review of systematic reviews do not suggest any benefit of using exercise therapy in the acute phase of LBP. None of the exercise types resulted in any effects, in any of the comparisons, which exceeded the established minimal important difference for pain and function [37]. This was true both when compared with placebo (sham ultrasound), with less strenuous interventions (advice to stay active, reassurance of an optimistic prognosis, and educational booklet), and with other forms of exercise therapy. This is important knowledge that the physiotherapist and general practitioner need to adopt in their clinical practice. Exercise is still used by physiotherapists for acute LBP, although not to as great extent as for subacute or chronic LBP [9]. Our findings imply that physiotherapists and general practitioners should be more reluctant in providing exercise therapy for acute LBP, and instead more strongly stress the good prognosis and provide reassurance and advice to stay active. They also need to communicate this knowledge to their patients with acute LBP so that patient and therapist can make an informed treatment decision together. Good patient-therapist communication is essential to achieve a collaborative rehabilitation and engage patients in their treatment [98]. In accordance with the principles of evidence-based practice, the physiotherapist and general practitioner should integrate their patient's preferences and values with their own clinical expertise and the research findings, to determine if and when exercise therapy could, or should, be the intervention of choice.

\section{Future research}

This systematic review of systematic reviews reveals many areas in which there is room for improvement in terms of rigorous conduct of RCTs that would enhance certainty of the evidence. Such improvement is necessary if we are to come to a more certain conclusion regarding the effects or non-effects of exercise therapy for acute LBP. Increasing sample size to reach sufficient power, standardizing outcomes and outcome measures, choosing relevant time points and relevant comparisons, and improving the reporting of conflicts of interest are some issues that would strengthen the certainty of evidence. Presenting study findings with minimally important differences and confidence intervals would enhance applicability of the findings.

Assigning more weight to results from studies with low risk of bias or excluding studies with high risk of bias are strategies that could resolve discrepancies in existing reviews and their included RCTs. Our systematic review found more systematic reviews than RCTs. In view of the many systematic reviews published and the large extent of overlap, the need to conduct another systematic review is limited, unless new RCTs are published. However, it is questionable whether it would be worthwhile to invest public funding in new trials on exercise therapy for acute LBP. Small trials with a high risk of bias are expected to overestimate the true effect. If 24 systematic reviews and 21 RCTs do not show a clinically important effect of exercise therapy for acute LBP, scarce resources for research might be better spent on prevention or treatment of chronic LBP. Reducing the enormous burden of chronic LBP seems a priority world-wide [99].

We found the GRADE approach challenging to apply and believe it would benefit from further development and guidance for use in systematic reviews of systematic reviews, to facilitate assessment of the certainty of the evidence. GRADE was developed to assess certainty of evidence based on risk of bias and other criteria in primary studies, whereas in a systematic review of systematic reviews, the unit of analysis is the included reviews. Methodological quality of the underlying RCTs is an important component of the GRADE assessment, and we had to rely on satisfactory quality appraisal and reporting by the review authors. However, we found considerable variation in the quality appraisal, and hence GRADE assessment, among the included reviews. We attempted to use Pollocks et al.'s algorithm for assigning GRADE levels [100], but did not find it suitable for this 
systematic review of systematic reviews. Greater consistency is needed with regard to how systematic review authors extract and present data and assess evidence, so that systematic review authors can rely on the underpinning data without having to go back to the original RCTs. Using GRADE for each outcome, time point, and intervention is feasible as long as the overlap is controlled in systematic reviews of systematic reviews.

\section{Ethical considerations}

When more systematic reviews than RCTs are conducted in a certain field, we need to consider other approaches to get answers. Doing more underpowered or biased RCTs is likely to further increase inconsistency and heterogeneity. Comparing one intervention to another, where none of the interventions have any superior effect compared with no treatment or sham, will not increase the certainty of evidence. Maybe the right question to ask is why large RCTs are still missing, despite three decades of systematic reviews based on RCTs? Faas et al. (74) studied 493 participants in 1993 and since then, no other RCT has succeeded in matching this number of participants. Why? Patients are often the ones who participate in the underpowered and biased studies and in the end the ones who receive the interventions. It does not seem appropriate or ethical to continue including patients in trials that will not adequately answer the research question.

\section{Conclusions}

The findings of this systematic review of systematic reviews suggest that there is very low-to-moderate certainty evidence that exercise therapy of any type may result in little or no important difference in pain or disability in adult patients with acute LBP, compared with other interventions, at any of the follow-up points reported. It is uncertain whether stabilization exercise in the acute phase reduces the risk of recurrence. Contradictory findings were seen in some small RCTs of low methodological quality. Adverse effects seem rare, but the total sample is too small to draw firm conclusions.

Knowledge about the certainty of evidence for the effectiveness of exercise therapy is important for the physiotherapist in clinical primary care practice and should be used to inform treatment decisions. The knowledge should be communicated to the patient, together with other treatment options, so that a fully informed, joint decision about treatment can be made.

\section{Supplementary information}

Supplementary information accompanies this paper at https://doi.org/10. 1186/s13643-020-01412-8.

Additional file 1. PRISMA checklist.

Additional file 2. Search strategies.
Additional file 3. Excluded systematic reviews.

Additional file 4. Summary of Findings Tables 4-12.

\section{Abbreviations}

Acute LBP: Acute low back pain; AMSTAR: A MeaSurement Tool to Assess systematic Reviews; CCA: Corrected covered area; MID: Minimal important difference; NRS: Numeric Rating Scale; NSAID: Non-steroid anti-inflammatory drug; ODI: Oswestry Disability Index; PICOS: Patient, Intervention,

Comparison/Control, Outcome, Setting/Study design;

PROSPERO: International prospective register of systematic reviews; PRISMA: Preferred Reporting Items for Systematic Review and Meta-Analysis Protocols; RMDQ: Roland Morris Disability Index; SoF: Summary of findings; VAS: Visual Analogue Scale

\section{Acknowledgements}

The authors wish to thank Sofia Tranaeus for commenting on an earlier draft of the manuscript, and statistician Marcus Praetorius Björk for help with the meta-analyses.

\section{Authors' contributions}

MK conceived the idea of this systematic review of systematic reviews and developed the protocol. He led the systematic review process and drafted the manuscript. SB and $A B$ contributed in every step of the review process and creation of the protocol. ML and LN contributed in the inclusion process, pilot testing of AMSTAR, third reviewer assistance in the AMSTAR assessment process, and in the GRADE assessment. MvT contributed recommendations for the review protocol and provided methodological guidance for the review process and the result synthesis. All authors were involved in interpretation of results, and read and approved the final manuscript. MK will consider when and if the need for future updates arise.

\section{Funding}

The first author received grant support from the Local Research and Development Board Fyrbodal, Region Västra Götaland, grant no. VGFOUFBD709601. Open access funding provided by University of Gothenburg.

\section{Availability of data and materials}

All data supporting the findings of this systematic review are included in this published article and its supplementary files.

Ethics approval and consent to participate

Not applicable.

\section{Consent for publication}

Not applicable.

\section{Competing interests}

The authors declare that they have no competing interests. MvT was first author or co-author in several systematic reviews included and excluded in this systematic review. However, MvT was not involved in the process of including or excluding systematic reviews, nor did he assess the methodological quality of the included systematic reviews.

\footnotetext{
Author details

${ }^{1}$ Region Skåne, Healthcare Centre Oxie, Malmö, Sweden. '2Department of Health and Rehabilitation, Unit of Physiotherapy, The Sahlgrenska Academy, Institute of Neuroscience and Physiology, University of Gothenburg, Gothenburg, Sweden. ${ }^{3}$ Region Västra Götaland, Närhälsan Uddevalla Rehabilitation, Uddevalla, Sweden. ${ }^{4}$ Region Västra Götaland, Research and Development Primary Health Care, Kungsgatan 12, 6th floor, SE-412 19 Gothenburg, Sweden. ${ }^{5}$ Department of Health Sciences, Faculty of Science, Amsterdam Movement Sciences, Vrije Universiteit Amsterdam, Amsterdam, the Netherlands. ${ }^{6}$ Department Physiotherapy \& Occupational Therapy, Aarhus University Hospital and Aarhus University, Aarhus, Denmark.
} 
Received: 20 November 2019 Accepted: 21 June 2020 Published online: 14 August 2020

\section{References}

1. Hoy D, March L, Brooks P, Blyth F, Woolf A, Bain C, et al. The global burden of low back pain: estimates from the global burden of Disease 2010 study. Ann Rheum Dis. 2014;73(6):968.

2. Mclntosh G, Hall H. Low back pain (acute). BMJ Clin Evid. 2011;2011:1102.

3. Olafsson G, Jonsson E, Fritzell P, Hägg O, Borgström F. A health economic lifetime treatment pathway model for low back pain in Sweden. J Med Econ. 2017:1-9.

4. GBD 2017 Disease and Injury Incidence and Prevalence Collaborators. Global, regional, and national incidence, prevalence, and years lived with disability for 354 diseases and injuries for 195 countries and territories, 19902017: a systematic analysis for the Global Burden of Disease study 2017. Lancet. 2018;392(10159):1789-858 London.

5. Pengel LH, Herbert RD, Maher CG, Refshauge KM. Acute low back pain: systematic review of its prognosis. BMJ (Clinical research ed). 2003; 327(7410):323.

6. Downie AS, Hancock MJ, Rzewuska M, Williams CM, Lin CW, Maher CG. Trajectories of acute low back pain: a latent class growth analysis. Pain. 2016;157(1):225-34.

7. SBU. Preventiva insatser vid akut smärta från rygg och nacke Stockholm: Statens beredning för medicinsk och social utvärdering (SBU) [The Swedish Agency for Health Technology Assessment and Assessment of Social Services]; 2016.

8. Chou R, Deyo R, Friedly J, Skelly A, Hashimoto R, Weimer M, Fu R, et al. Noninvasive treatments for low back pain. In: Comparative effectiveness review no. 169. (prepared by the Pacific northwest evidence-based practice center under contract no. 290-2012-00014-I.) AHRQ publication no. 16EHC004- EF. Rockville: Agency for Healthcare Research and Quality; 2016.

9. Bernhardsson S, Oberg B, Johansson K, Nilsen P, Larsson ME. Clinical practice in line with evidence? A survey among primary care physiotherapists in western Sweden. J Eval Clin Pract. 2015;21(6):1169-77.

10. Nordeman L, Nilsson B, Moller M, Gunnarsson R. Early access to physical therapy treatment for subacute low back pain in primary health care: a prospective randomized clinical trial. Clin J Pain. 2006;22(6):505-11.

11. Keating JL, McKenzie JE, O'Connor DA, French S, Walker BF, Charity M, et al. Providing services for acute low-back pain: a survey of Australian physiotherapists. Man Ther. 2016;22(Supplement C):145-52.

12. Wong JJ, Cote $\mathrm{P}$, Sutton DA, Randhawa $\mathrm{K}, \mathrm{Yu} \mathrm{H}$, Varatharajan S, et al. Clinical practice guidelines for the noninvasive management of low back pain: a systematic review by the Ontario protocol for traffic Injury management (OPTIMa) collaboration. Eur J Pain (London, England). 2017;21(2):201-16.

13. Koes BW, van Tulder M, Lin CW, Macedo LG, McAuley J, Maher C. An updated overview of clinical guidelines for the management of non-specific low back pain in primary care. Eur Spine J. 2010;19(12):2075-94.

14. Ladeira CE. Evidence based practice guidelines for management of low back pain: physical therapy implications. Rev Bras Fis. 2011;15(3):190-9.

15. Chou R, Hoffman LH. Guideline for the evaluation and management of low back pain - evidence review. Glenview: American Pain Society; 2017

16. Delitto A, George SZ, Van Dillen LR, Whitman JM, Sowa G, Shekelle P, et al. Low back pain. J Orthop Sports Phys Ther. 2012;42(4):A1-57.

17. Macedo LG, Saragiotto BT, Yamato TP, Costa LO, Menezes Costa LC, Ostelo RW, et al. Motor control exercise for acute non-specific low back pain. Cochrane Database Syst Rev. 2016;2:Cd012085.

18. Chou R, Deyo R, Friedly J, Skelly A, Hashimoto R, Weimer M, et al. Nonpharmacologic therapies for low back pain: a systematic review for an American College of Physicians Clinical Practice Guideline. Ann Intern Med. 2017;166(7):493-505.

19. Sackett DL, Rosenberg WM, Gray JA, Haynes RB, Richardson WS. Evidence based medicine: what it is and what it isn't. BMJ (Clinical Research Ed). 1996;312(7023):71-2

20. Hidalgo B, Detrembleur $C$, Hall T, Mahaudens $P$, Nielens $H$. The efficacy of manual therapy and exercise for different stages of non-specific low back pain: an update of systematic reviews. J Man Manip Ther. 2014;22(2):59-74.

21. Nijs J, Van Houdenhove B. From acute musculoskeletal pain to chronic widespread pain and fibromyalgia: application of pain neurophysiology in manual therapy practice. Man Ther. 2009;14(1):3-12.

22. Wenger HC, Cifu AS. Treatment of low back pain. Jama. 2017;318(8):743-4.
23. Rubinstein SM, Terwee CB, Assendelft WJ, de Boer MR, van Tulder MW. Spinal manipulative therapy for acute low-back pain. Cochrane Database Syst Rev. 2012;2012(9):Cd008880.

24. Franke H, Fryer G, Ostelo RW, Kamper SJ. Muscle energy technique for nonspecific low-back pain. Cochrane Database Syst Rev. 2015;(2):Cd009852.

25. Saragiotto BT, Machado GC, Ferreira ML, Pinheiro MB, Abdel Shaheed C, Maher CG. Paracetamol for low back pain. Cochrane Database Syst Rev. 2016;(6):Cd012230.

26. Fritz JM, Magel JS, McFadden M, Asche C, Thackeray A, Meier W, et al. Early physical therapy vs usual care in patients with recent-onset low back pain: a randomized clinical trial. JAMA. 2015;314(14):1459-67.

27. Machado LAC, Maher CG, Herbert RD, Clare H, McAuley JH. The effectiveness of the McKenzie method in addition to first-line care for acute low back pain: a randomized controlled trial. BMC Med. 2010;8:10.

28. Rackwitz B, de Bie R, Limm H, von Garnier K, Ewert T, Stucki G. Segmental stabilizing exercises and low back pain. What is the evidence? A systematic review of randomized controlled trials [with consumer summary]. Clin Rehabil. 2006;20(7):553-67.

29. Shamseer L, Moher D, Clarke M, Ghersi D, Liberati A, Petticrew M, et al. Preferred reporting items for systematic review and meta-analysis protocols (PRISMA-P) 2015: elaboration and explanation. BMJ (Clinical Research ed). 2015;349:97647.

30. Smith V, Devane D, Begley CM, Clarke M. Methodology in conducting a systematic review of systematic reviews of healthcare interventions. BMC Med Res Methodol. 2011;11(1):15.

31. Pollock M, Fernandes RM, Becker LA, Pieper D, Hartling L. Chapter V: overviews of reviews. In: JPT H, Thomas J, Chandler J, Cumpston M, Li T, Page MJ, Welch VA, Cochrane handbook for systematic reviews of interventions version 6.0 (updated March 2020). Cochrane. p. 2020. Available from www.training.cochrane.org/handbook.

32. Lunny C, Brennan SE, McDonald S, McKenzie JE. Toward a comprehensive evidence map of overview of systematic review methods: paper 1-purpose, eligibility, search and data extraction. Syst Rev. 2017;6(1):231.

33. Lunny C, Brennan SE, McDonald S, McKenzie JE. Toward a comprehensive evidence map of overview of systematic review methods: paper 2-risk of bias assessment; synthesis, presentation and summary of the findings; and assessment of the certainty of the evidence. Syst Rev. 2018;7(1):159.

34. Moher D, Liberati A, Tetzlaff J, Altman DG. Preferred reporting items for systematic reviews and meta-analyses: the PRISMA statement. Int J Surg (London, England). 2010;8(5):336-41.

35. Exercise Therapy [Internet]. 2017. [cited 11 Oct 2017]. Available from: https:// www.ncbi.n/m.nih.gov/mesh/?term=exercise+therapy .

36. The McKenzie Institute International. 2017. [Cited 26 June 2020]. Available from: http://www.mckenzieinstitute.org/clinicians/research-and-resources/ reference-list/lumbar\%3A-systematic-reviews/.

37. Ostelo RW, Deyo RA, Stratford P, Waddell G, Croft P, Von Korff M, et al, Interpreting change scores for pain and functional status in low back pain: towards international consensus regarding minimal important change. Spine (Phila Pa 1976). 2008;33(1):90-4

38. Higgins JPT, Thomas J, Chandler J, Cumpston M, Li T, Page MJ, Welch VA, editors. Cochrane Handbook for Systematic Reviews of Interventions version 6.0 (updated July 2019): Cochrane; 2019. Available from: Address=www. training.cochrane.org/handbook.

39. Pieper D, Antoine SL, Mathes T, Neugebauer EA, Eikermann M. Systematic review finds overlapping reviews were not mentioned in every other overview. J Clin Epidemiol. 2014;4(67):368-75.

40. Shea BJ, Bouter LM, Peterson J, Boers M, Andersson N, Ortiz Z, et al. External validation of a measurement tool to assess systematic reviews (AMSTAR). PLoS One. 2007:2(12):e1350.

41. Shea BJ, Grimshaw JM, Wells GA, Boers M, Andersson N, Hamel C, et al. Development of AMSTAR: a measurement tool to assess the methodological quality of systematic reviews. BMC Med Res Methodol. 2007;7:10.

42. Shea BJ, Reeves BC, Wells G, Thuku M, Hamel C, Moran J, et al. AMSTAR 2: a critical appraisal tool for systematic reviews that include randomised or non-randomised studies of healthcare interventions, or both. BMJ. 2017;358: j4008.

43. Higgins JPT, Deeks JJ. Chapter 7: Selecting studies and collecting data. Cochrane collaboration. 2008. Available from: www.cochrane-handbook.org

44. GRADEpro GDT: GRADEpro Guideline Development Tool [Software]. [cited 26 June 2020]. McMaster University, (developed by Evidence Prime, Inc.) 2015. Available from: https://gradepro.org 
45. Doi SA, Barendregt JJ, Khan S, Thalib L, Williams GM. Advances in the metaanalysis of heterogeneous clinical trials I: the inverse variance heterogeneity model. Contemp Clin Trials. 2015:45(Pt A):130-8.

46. MetaXL User Guide. Version 5.3. Jan J Barendregt, Suhail A Doi. (C) EpiGear International Pty Ltd. ABN 51134897 411. Sunrise Beach, Queensland, Australia, 2011-2016.

47. Guyatt GH, Oxman AD, Kunz R, Brozek J, Alonso-Coello P, Rind D, et al. GRADE guidelines 6. Rating the quality of evidence--imprecision. J Clin Epidemiol. 2011;64(12):1283-93.

48. Hultcrantz M, Rind D, Akl EA, Treweek S, Mustafa RA, lorio A, et al. The GRADE working group clarifies the construct of certainty of evidence. J Clin Epidemiol. 2017;87:4-13.

49. Koes BW, Bouter LM, Beckerman H, van der Heijden G, Knipschild PG. Physiotherapy exercises and back pain: a blinded review. BMJ. 1991; 302(6792):1572-6.

50. Farrell JP, Twomey LT. Acute low back pain. Comparison of two conservative treatment approaches. Med J Austr. 1982;1(4):160-4

51. Waterworth RH, IA. An open study of diflunisal, conservative and manipulative therapy in the management of acute mechanical low back pain. N Z Med J. 1985;98(779):372-5.

52. Gilbert JR, Taylor DW, Hildebrand A, Evans C. Clinical trial of common treatments for low back pain in family practice. BMJ (Clinical research ed). 1985;291:791-4.

53. Stankovic R, Johnell O. Conservative treatment of acute low-back pain. A prospective randomized trial: McKenzie method of treatment versus patient education in "mini back school". Spine. 1990;15(2):120-3.

54. Roberts A. The conservative treatment of low back pain: a study of McKenzie physiotherapy and slow release ketoprofen [DM thesis]: University of Nottingham; 1991.

55. Erhard RE, Delitto A, Cibulka MT. Relative effectiveness of an extension program and a combined program of manipulation and flexion and extension exercises in patients with acute low back syndrome. Phys Ther. 1994;74(12):1093-100.

56. Schenk R, Dionne C, Simon C, Johnson R. Effectiveness of mechanical diagnosis and therapy in patients with back pain who meet a clinical prediction rule for spinal manipulation. J Man Manip Ther. 2012;20(1):43-9.

57. Faas A, Battie MC, Malmivaara A. Exercises: which ones are worth trying, for which patients, and when? Spine. 1996;21(24):2874-9.

58. Delitto A, Cibulka MT, Erhard RE, Bowling RW, Tenhula JA. Evidence for use of an extension-mobilization category in acute low back syndrome: a prescriptive validation pilot study. Phys Ther. 1993;73(4):216-22 discussion 23-8.

59. Faas A, Chavannes AW, van Eijk JT, Gubbels JW. A randomized, placebocontrolled trial of exercise therapy in patients with acute low back pain. Spine. 1993;18(11):1388-95.

60. Malmivaara A, Hakkinen U, Aro T, Heinrichs ML, Koskenniemi L, Kuosma E, et al. The treatment of acute low back pain - bed rest, exercises, or ordinary activity? N Engl J Med. 1995;332(6):351-5.

61. Dettori JR, Bullock SH, Sutlive TG, Franklin RJ, Patience T. The effects of spinal flexion and extension exercises and their associated postures in patients with acute low back pain. Spine. 1995;20(21):2303-12.

62. Keller A, Hayden J, Bombardier C, van Tulder M. Effect sizes of non-surgical treatments of non-specific low-back pain. Eur Spine J. 2007;16(11):1776-88

63. van Tulder MW, Koes BW, Bouter LM. Conservative treatment of acute and chronic nonspecific low back pain. A systematic review of randomized controlled trials of the most common interventions. Spine (Phila Pa 1976). 1997;22(18):2128-56

64. van Tulder MW, Malmivaara A, Esmail R, Koes BW. Exercise therapy for low back pain. Cochrane Database Syst Rev. 2000;(2):Cd000335.

65. Cherkin DC, Deyo RA, Battie M, Street J, Barlow W. A comparison of physica therapy, chiropractic manipulation, and provision of an educational booklet for the treatment of patients with low back pain. N Engl J Med. 1998; 339(15):1021-9.

66. Seferlis T, Nemeth $\mathrm{G}$, Carlsson AM, Gillstrom P. Conservative treatment in patients sick-listed for acute low-back pain: a prospective randomised study with 12 months' follow-up. Eur Spine J. 1998;7(6):461-70.

67. Underwood MR, Morgan J. The use of a back class teaching extension exercises in the treatment of acute low back pain in primary care. Fam Pract. 1998;15(1):9-15.

68. Ferreira PH, Ferreira ML, Maher CG, Herbert RD, Refshauge K. Specific stabilisation exercise for spinal and pelvic pain: a systematic review. Aust $J$ Physiother. 2006;52(2):79-88.
69. Hayden JA, van Tulder MW, Malmivaara A, Koes BW. Exercise therapy for treatment of non-specific low back pain (Cochrane review) [with consumer summary]. Cochrane Database Syst Rev. 2005;3.

70. Clare HA, Adams R, Maher CG. A systematic review of efficacy of McKenzie therapy for spinal pain. Aust J Physiother. 2004;50(4):209-16.

71. Kriese M, Clijsen R, Taeymans J, Cabri J. Segmental stabilization in low back pain: a systematic review. Sportverletz Sportschaden. 2010;24(1):17-25.

72. Hides JA, Richardson CA, Jull GA. Multifidus muscle recovery is not automatic after resolution of acute, first-episode low back pain. Spine. 1996; 21(23):2763-9.

73. Ferreira ML, Ferreira PH, Latimer J, Herbert R, Maher CG. Efficacy of spinal manipulative therapy for low back pain of less than three months' duration. J Manip Physiol Ther. 2003;26(9):593-601.

74. Schenk RJ, Jozefczyk C, Kopf A. A randomized trial comparing interventions in patients with lumbar posterior derangement. J Man Manip Ther. 2003; 11(2):95-102.

75. Chok B, Lee R, Latimer J, Tan SB. Endurance training of the trunk extensor muscles in people with subacute low back pain. Phys Ther. 1999;79(11): 1032-42.

76. Machado LAC, de Souza MS, Ferreira PH, Ferreira ML. The McKenzie method for low back pain: a systematic review of the literature with a meta-analysis approach [with consumer summary]. Spine. 2006;31(9):E254-62.

77. Aluko A, DeSouza L, Peacock J. The effect of core stability exercises on variations in acceleration of trunk movement, pain, and disability during an episode of acute nonspecific low back pain: a pilot clinical trial. J Manip Physiol Ther. 2013;36(8):497-504 e1-3.

78. Hauggaard A, Persson AL. Specific spinal stabilisation exercises in patients with low back pain -- a systematic review. Phys Ther Rev. 2007;12(3):233-48.

79. Mayer JM, Ralph L, Look M, Erasala GN, Verna JL, Matheson LN, et al. Treating acute low back pain with continuous low-level heat wrap therapy and/or exercise: a randomized controlled trial. Spine J. 2005;5(4):395-403.

80. Liddle SD, Gracey JH, Baxter GD. Advice for the management of low back pain: a systematic review of randomised controlled trials. Man Ther. 2007; 12(4):310-27.

81. Engers $A$, Jellema $P$, Wensing $M$, van der Windt $D$, Grol R, van Tulder MW. Individual patient education for low back pain (Cochrane review) [with consumer summary]. Cochrane Database Syst Rev. 2008;1.

82. May S, Johnson R. Stabilisation exercises for low back pain: a systematic review. Physiotherapy. 2008;94(3):179-89.

83. Brennan GP, Fritz JM, Hunter SJ, Thackeray A, Delitto A, Erhard RE. Identifying subgroups of patients with acute/subacute "nonspecific" low back pain: results of a randomized clinical trial. Spine. 2006: 31(6):623-31

84. Ferreira MC, Penido $H$, Aun A, Ferreira P, Ferreira ML, Oliveira VC. Efficacy of motor control exercises for lumbopelvic pain: a systematic review. Fisioterapia e Pesquisa. 2009;16(4):374-9.

85. Choi BK, Verbeek JH, Tam WW, Jiang JY. Exercises for prevention of recurrences of low-back pain. Occup Environ Med. 2010;67(11):795-6.

86. Dahm KT, Brurberg KG, Jamtvedt G, Hagen KB. Advice to rest in bed versus advice to stay active for acute low-back pain and sciatica (Cochrane review) [with consumer summary]. Cochrane Database Syst Rev. 2010;6.

87. Dunsford A, Kumar S, Clarke S. Integrating evidence into practice: use of McKenzie-based treatment for mechanical low back pain. J Multidiscip Healthc. 2011:4:393-402.

88. Surkitt LD, Ford JJ, Hahne AJ, Pizzari T, McMeeken JM. Efficacy of directional preference management for low back pain: a systematic review [with consumer summary]. Phys Ther. 2012;92(5):652-65.

89. Lam OT, Strenger DM, Chan-Fee M, Pham PT, Preuss RA, Robbins SM. Effectiveness of the McKenzie method of mechanical diagnosis and therapy for treating low back pain: literature review with meta-analysis. J Orthop Sports Phys Ther. 2018;48(6):476-90.

90. Bickel CS, Cross JM, Bamman MM. Exercise dosing to retain resistance training adaptations in young and older adults. Med Sci Sports Exerc. 2011; 43(7):1177-87.

91. Halliday MH, Garcia AN, Amorim AB, Machado GC, Hayden JA, Pappas E, et al. Treatment effect sizes of mechanical diagnosis and therapy for pain and disability in patients with low back pain: a systematic review. J Orthop Sports Phys Ther. 2019;49(4):219-29.

92. Swinkels A, Cochrane K, Burt A, Johnson L, Lunn T, Rees AS. Exercise interventions for non-specific low back pain: an overview of systematic reviews. Phys Ther Rev. 2009;14(4):247-59. 
93. Maher C, Underwood M, Buchbinder R. Non-specific low back pain. Lancet (London, England). 2017;389(10070):736-47.

94. Oliveira CB, Maher CG, Pinto RZ, Traeger AC, Lin CC, Chenot JF, et al. Clinical practice guidelines for the management of non-specific low back pain in primary care: an updated overview. Eur Spine J. 2018;27(11):2791-803.

95. Stochkendahl MJ, Kjaer P, Hartvigsen J, Kongsted A, Aaboe J, Andersen M, et al. National Clinical Guidelines for non-surgical treatment of patients with recent onset low back pain or lumbar radiculopathy. Eur Spine J. 2018;27(1): $60-75$.

96. Rucker G, Schumacher M. Simpson's paradox visualized: the example of the rosiglitazone meta-analysis. BMC Med Res Methodol. 2008;8:34.

97. Jordan KP, Kadam UT, Hayward R, Porcheret M, Young C, Croft P. Annual consultation prevalence of regional musculoskeletal problems in primary care: an observational study. BMC Muscuoskelet Disord. 2010;11:144.

98. Bernhardsson S, Samsson KS, Johansson K, Öberg B, Larsson MEH. A preference for dialogue: exploring the influence of patient preferences on clinical decision making and treatment in primary care physiotherapy. Eur J Phys. 2019;21(2):107-14.

99. Hartvigsen J, Hancock MJ, Kongsted A, Louw Q, Ferreira ML, Genevay S, et al. What low back pain is and why we need to pay attention. Lancet (London, England). 2018;391(10137):2356-67.

100. Pollock A, Farmer SE, Brady MC, Langhorne P, Mead GE, Mehrholz J, et al. An algorithm was developed to assign GRADE levels of evidence to comparisons within systematic reviews. J Clin Epidemiol. 2016;70:106-10.

\section{Publisher's Note}

Springer Nature remains neutral with regard to jurisdictional claims in published maps and institutional affiliations.

Ready to submit your research? Choose BMC and benefit from:

- fast, convenient online submission

- thorough peer review by experienced researchers in your field

- rapid publication on acceptance

- support for research data, including large and complex data types

- gold Open Access which fosters wider collaboration and increased citations

- maximum visibility for your research: over $100 \mathrm{M}$ website views per year

At $\mathrm{BMC}$, research is always in progress.

Learn more biomedcentral.com/submissions 